Supporting Information

for

\title{
Organocatalytic Asymmetric Biginelli-like Reaction Involving Isatin
}

Mattia Stucchi, ${ }^{a}$ Giordano Lesma, ${ }^{a}$ Fiorella Meneghetti, ${ }^{b}$ Giulia Rainoldi, ${ }^{a}$ Alessandro Sacchetti ${ }^{\mathrm{c}}$ and Alessandra Silvani ${ }^{\mathrm{a}, *}$

${ }^{a}$ Università di Milano, Dipartimento di Chimica, via Golgi 19, Milano, 20133, Italy. ${ }^{b}$ Dipartimento di Scienze Farmaceutiche, Università degli Studi di Milano, via L. Mangiagalli 25, 20133 Milano, Italy. ${ }^{c}$ Politecnico di Milano, Dipartimento di Chimica, Materiali ed Ing.Chimica 'Giulio Natta', p.zza Leonardo da Vinci 32, Milano, 20133, Italy.

E-mail: alessandra.silvani@unimi.it

Table of contents

Copies of ${ }^{1} \mathrm{H}$ and ${ }^{13} \mathrm{C}$ NMR spectra S2-S16

(new compounds: 5a-j, 6, 7, 8, 9a, 9b)

HPLC chromatograms (compounds 5a-j)

$\mathrm{S} 17-\mathrm{S} 21$

Experimental for X-ray analysis (compound 5h)

Computational data

S25-S34 
Compound 5a: ${ }^{1} \mathrm{H}$ NMR (400 MHz, $\mathrm{CDCl}_{3}$ )

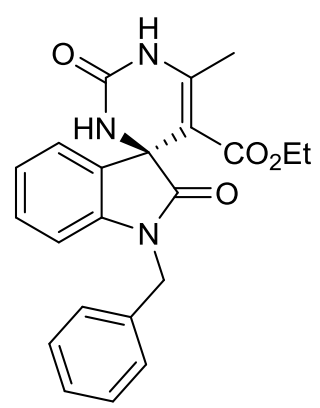

$5 a$

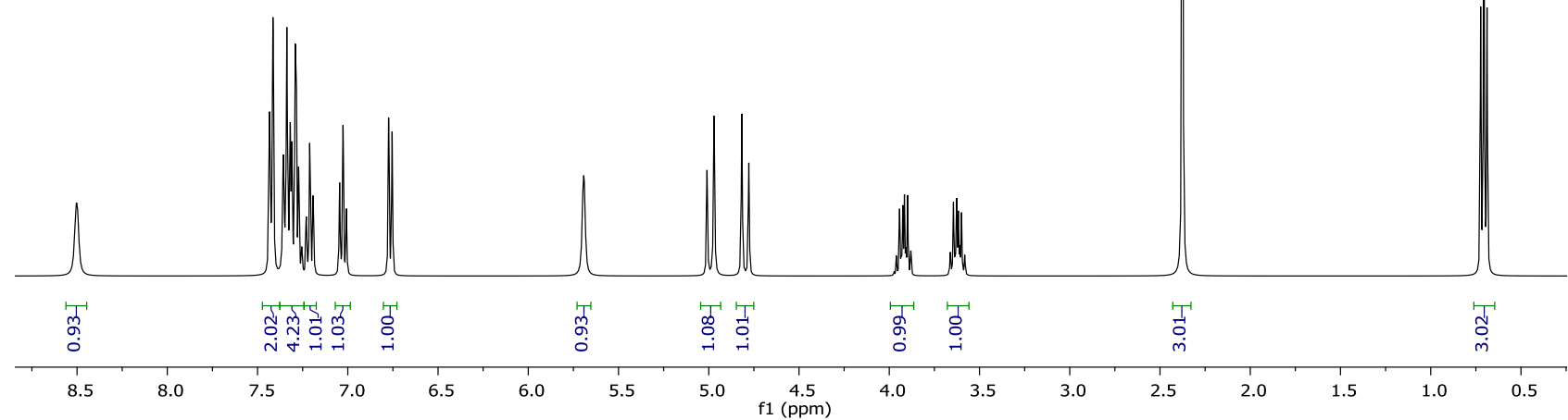

Compound 5a: ${ }^{13} \mathrm{C}$ NMR (100 MHz, $\mathrm{CDCl}_{3}$ )
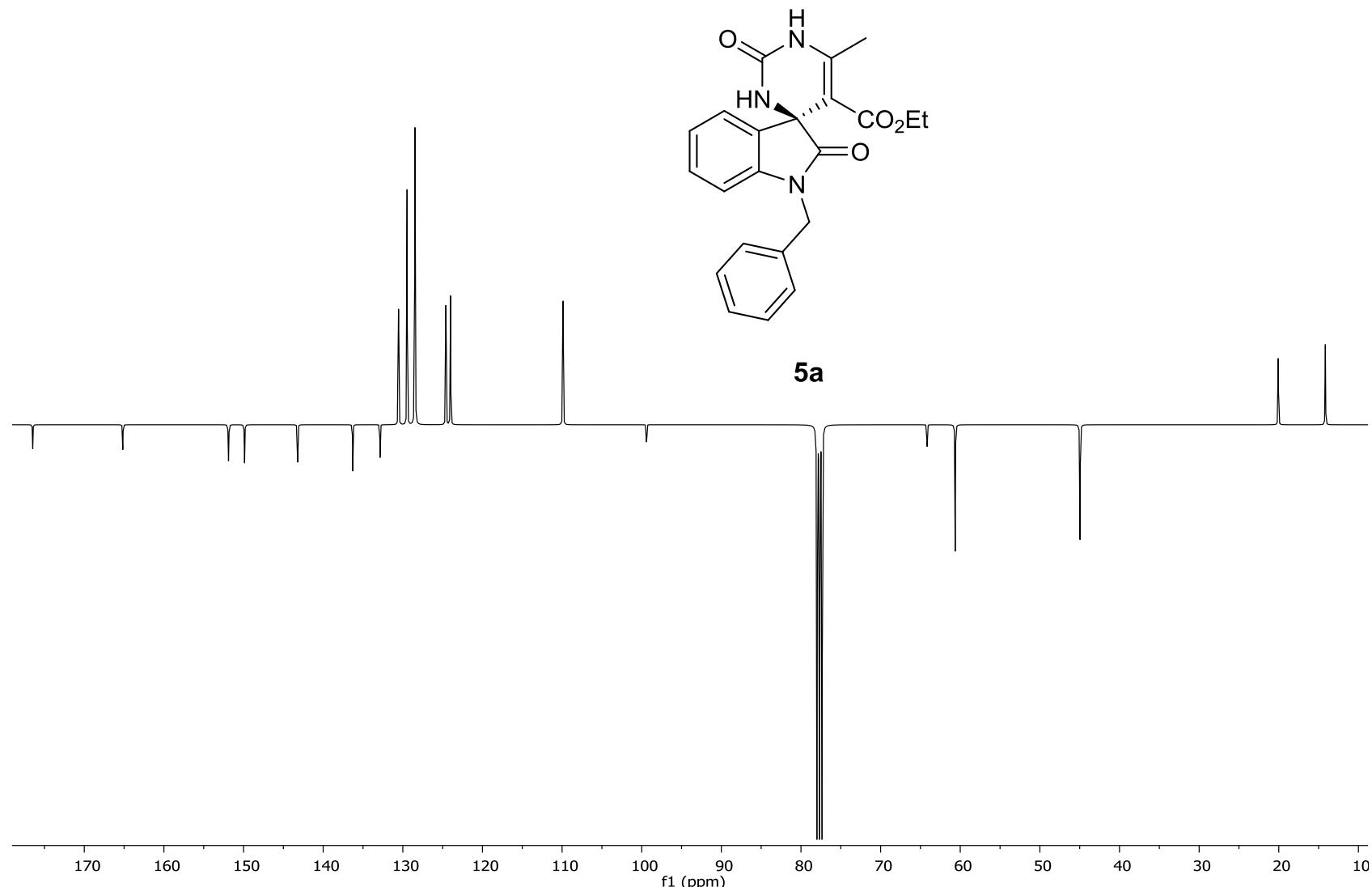
Compound 5b: ${ }^{1} \mathrm{H}$ NMR (300 MHz, $\mathrm{CDCl}_{3}$ )

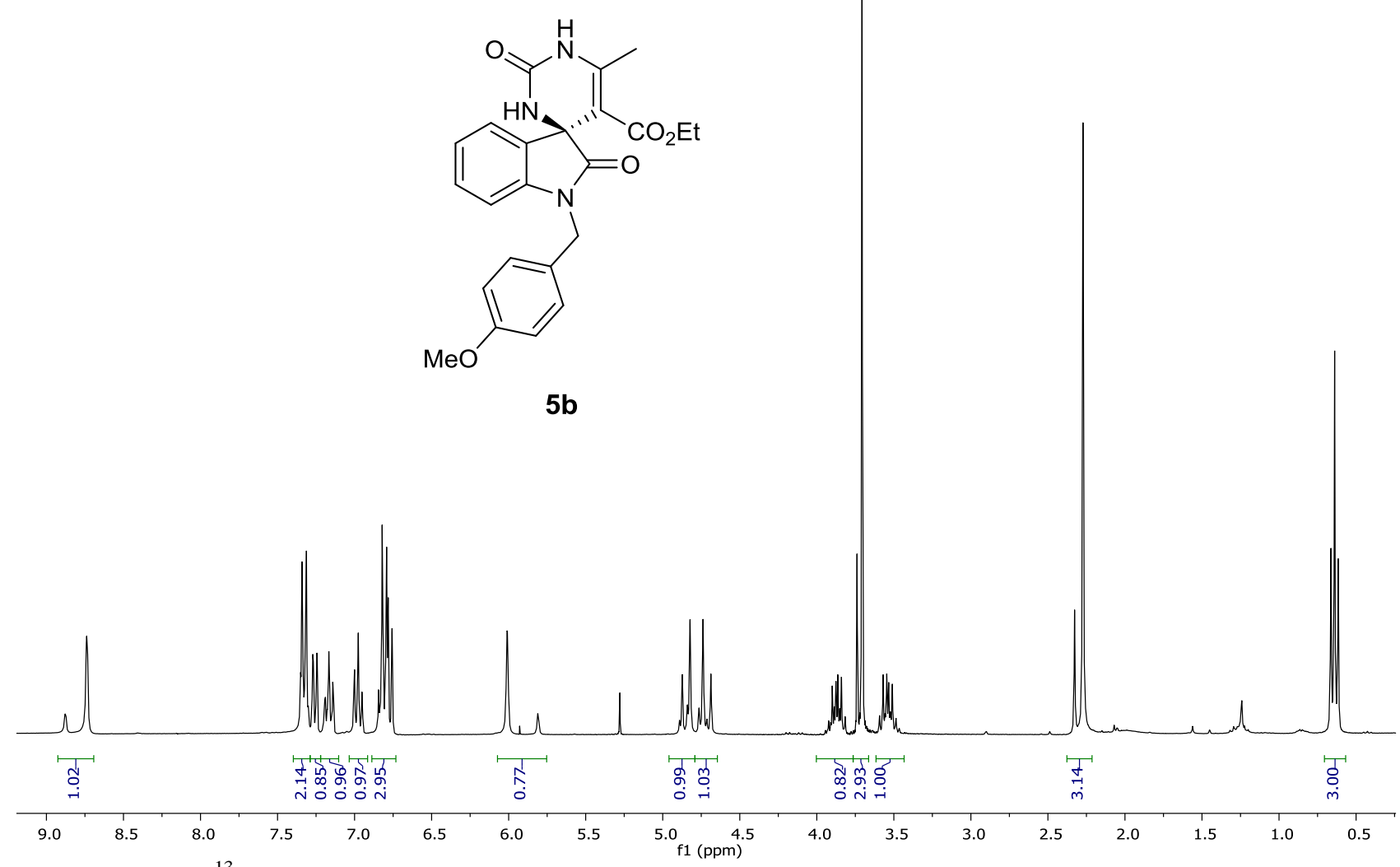

Compound 5b: ${ }^{13} \mathrm{C}$ NMR (75 MHz, $\mathrm{CDCl}_{3}$ )
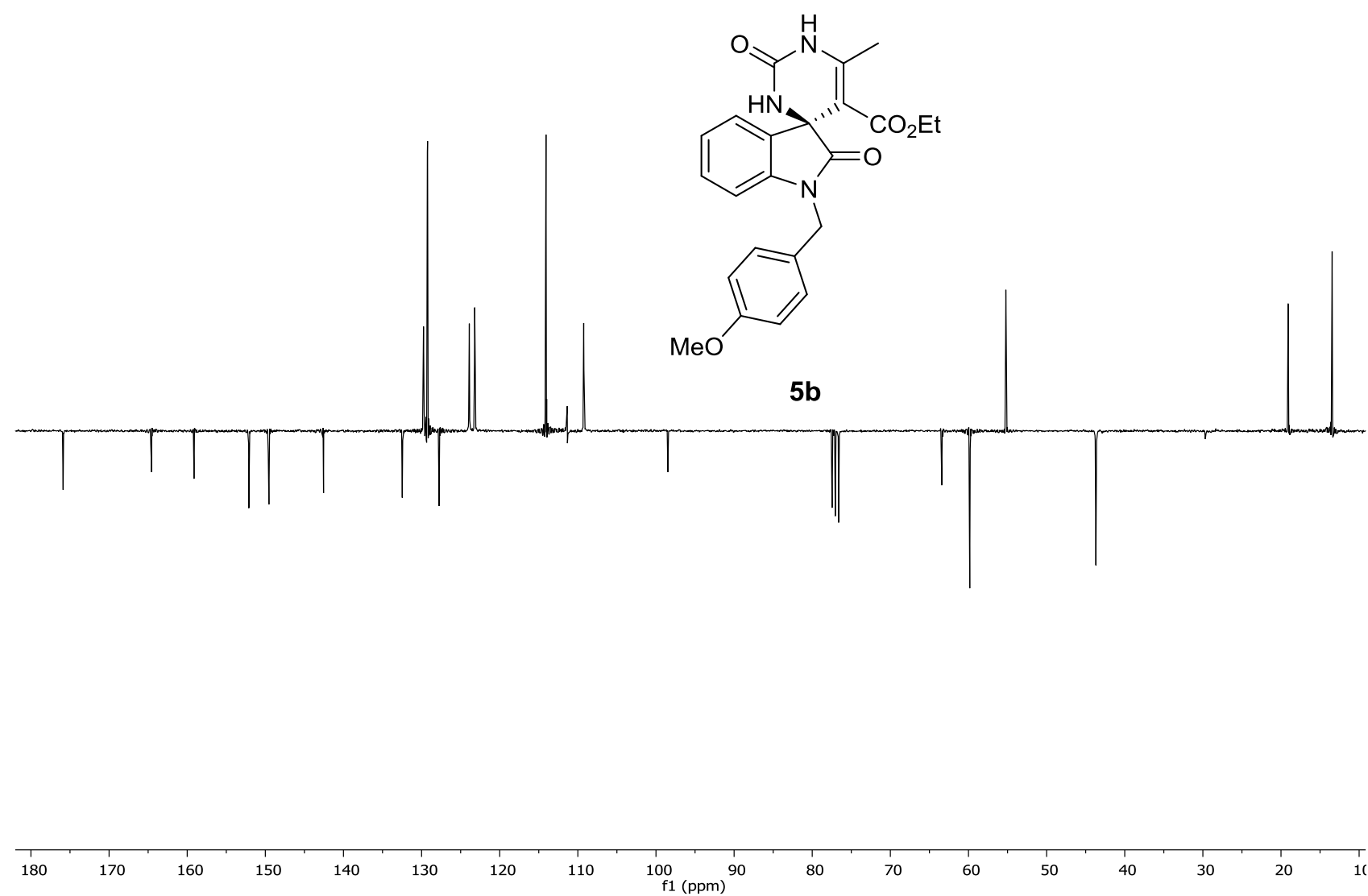
Compound 5c: ${ }^{1} \mathrm{H}$ NMR $\left(300 \mathrm{MHz}, \mathrm{CDCl}_{3}\right)$
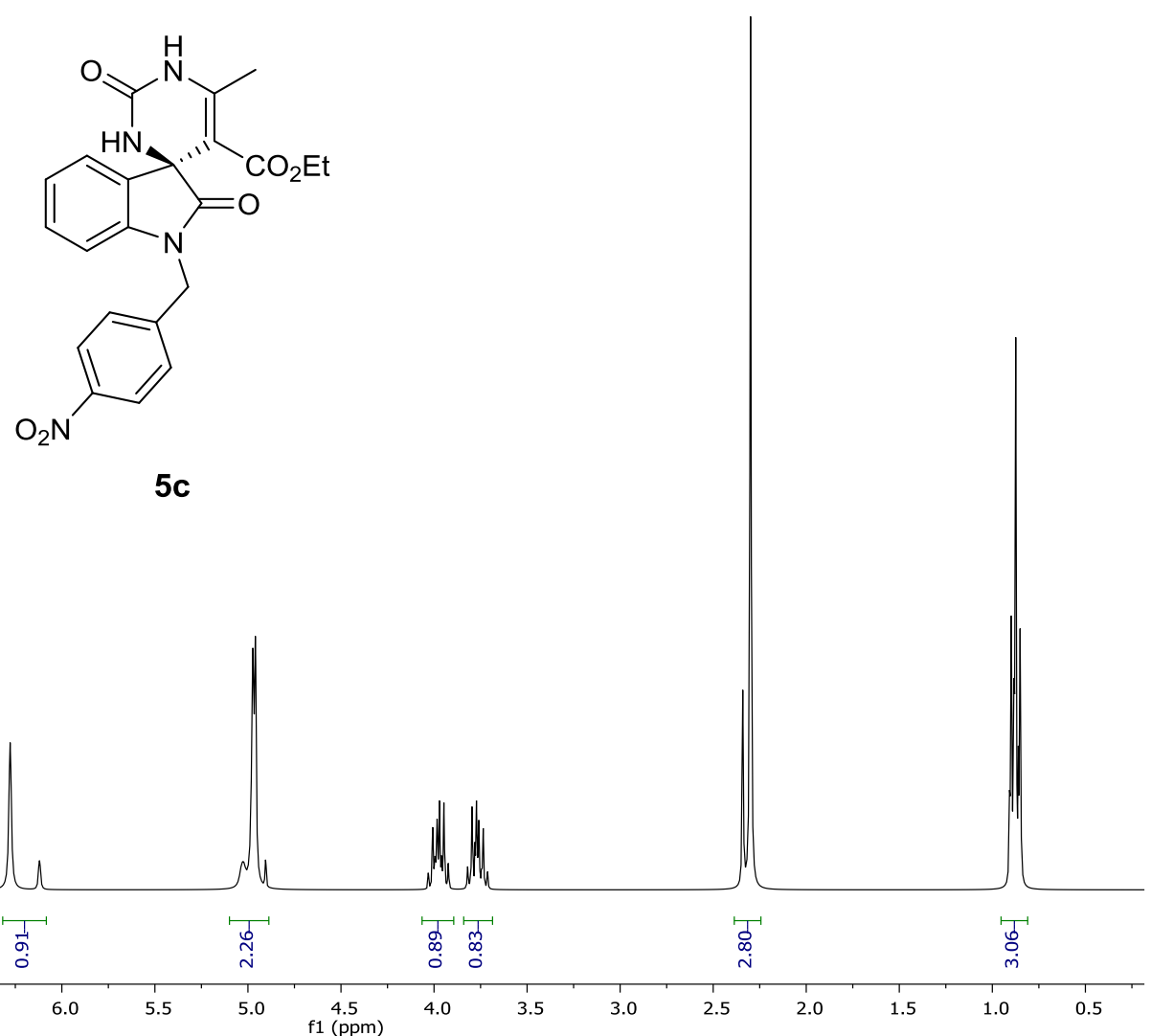

Compound 5c: ${ }^{13} \mathrm{C}$ NMR (75 MHz, $\mathrm{CDCl}_{3}$ )

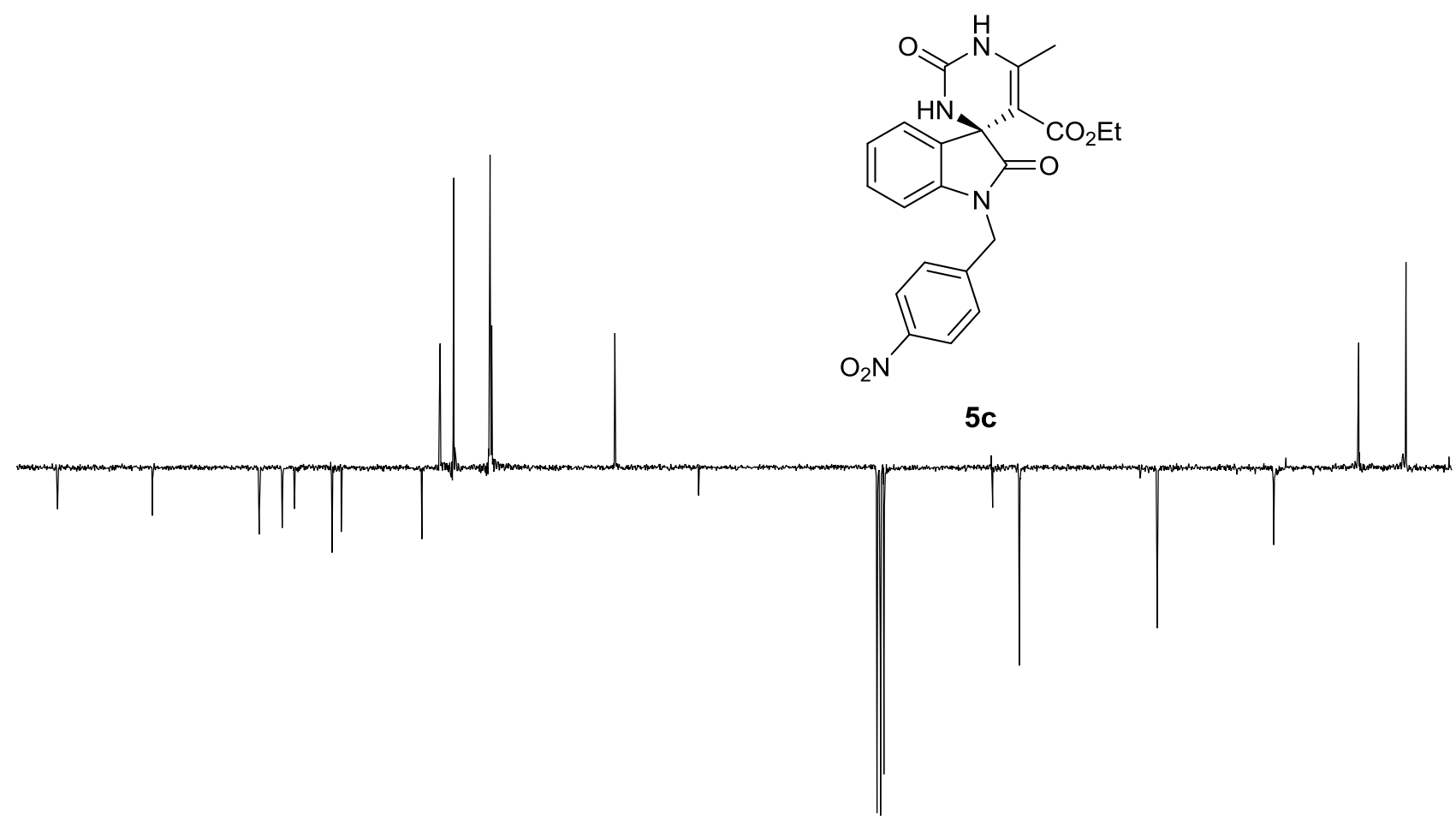

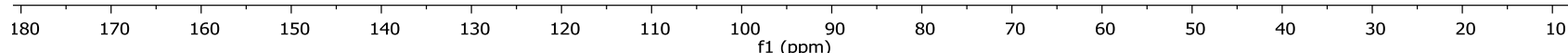


Compound 5d: ${ }^{1} \mathrm{H}$ NMR (400 MHz, DMSO- $d_{6}$ )

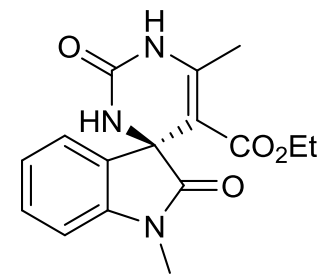

$5 d$

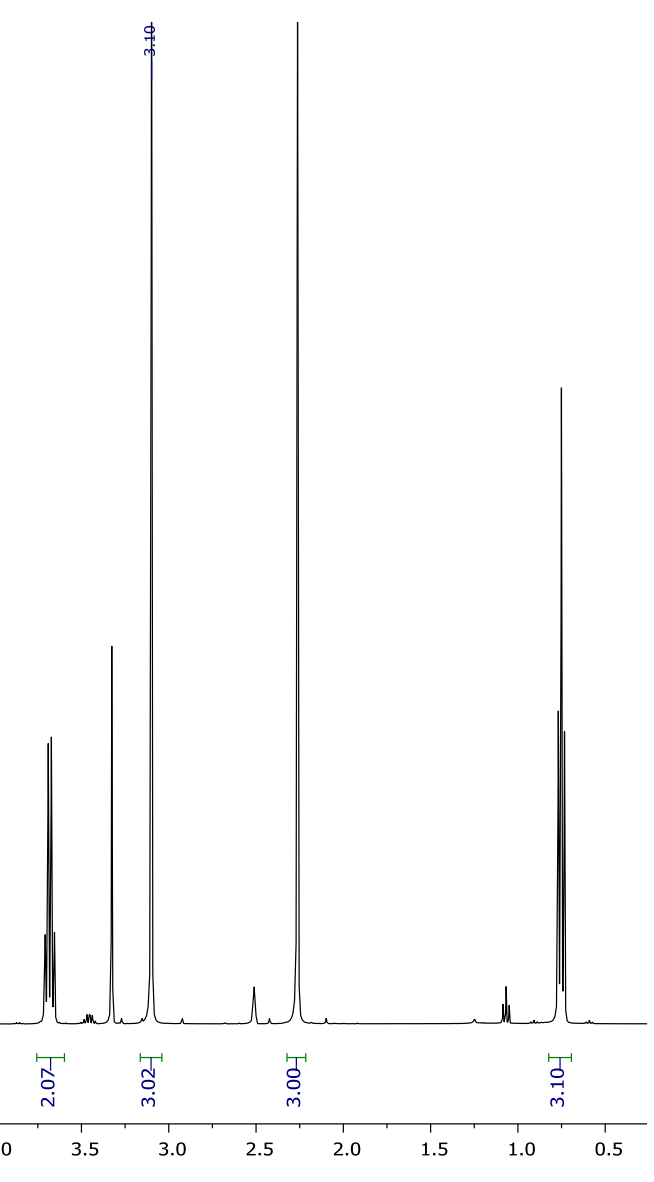

Compound 5d: ${ }^{13} \mathrm{C}$ NMR (100 MHz, DMSO- $d_{6}$ )
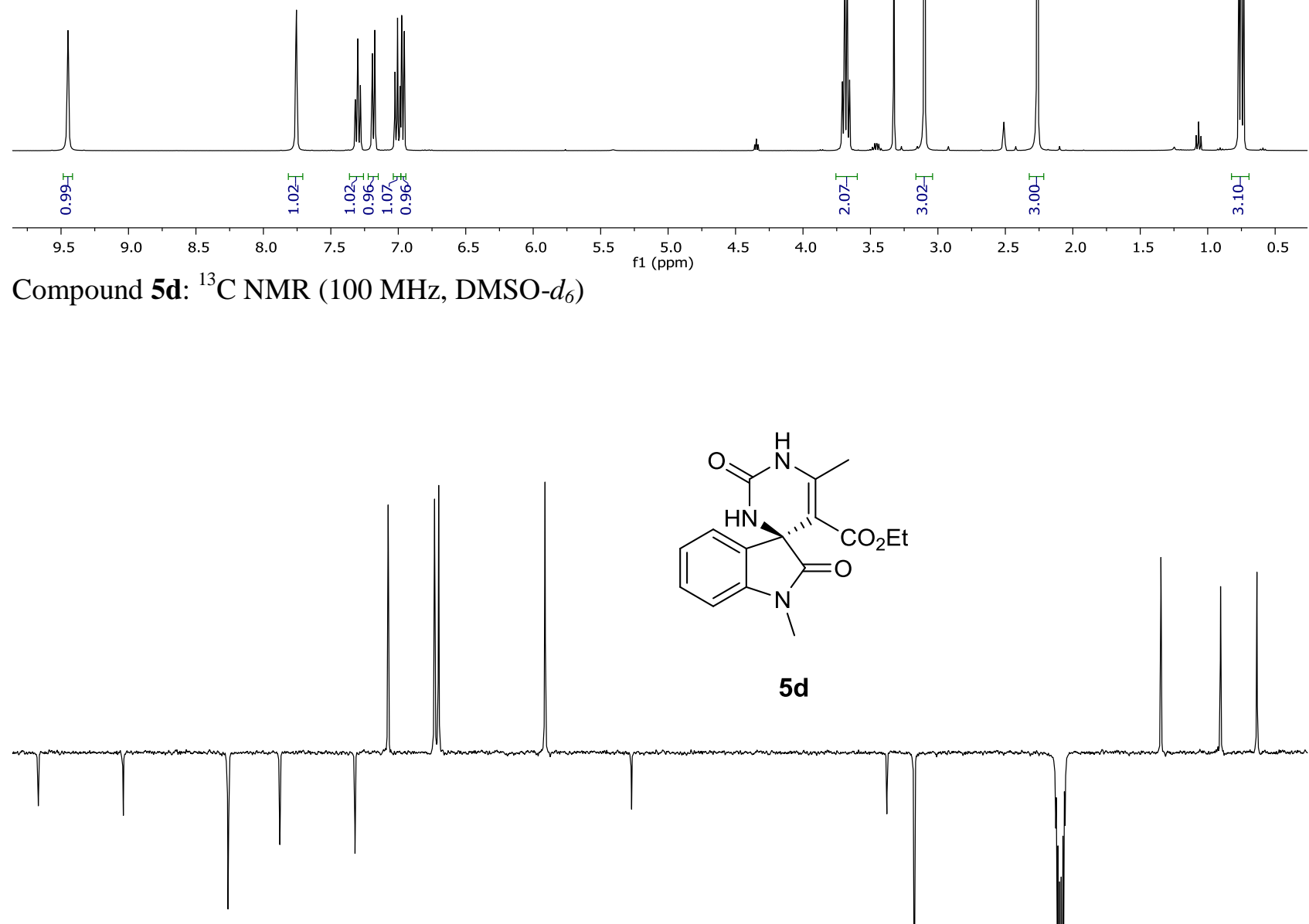

$170 \quad 160 \quad 150$

$140 \quad 130$

$130 \quad 120$

$20 \quad 110$

$100 \begin{array}{r}90 \\ \mathrm{f} 1(\mathrm{ppm})\end{array}$

$70 \quad 60$ 
Compound 5e: ${ }^{1} \mathrm{H}$ NMR (300 MHz, $\left.\mathrm{CDCl}_{3}\right)$
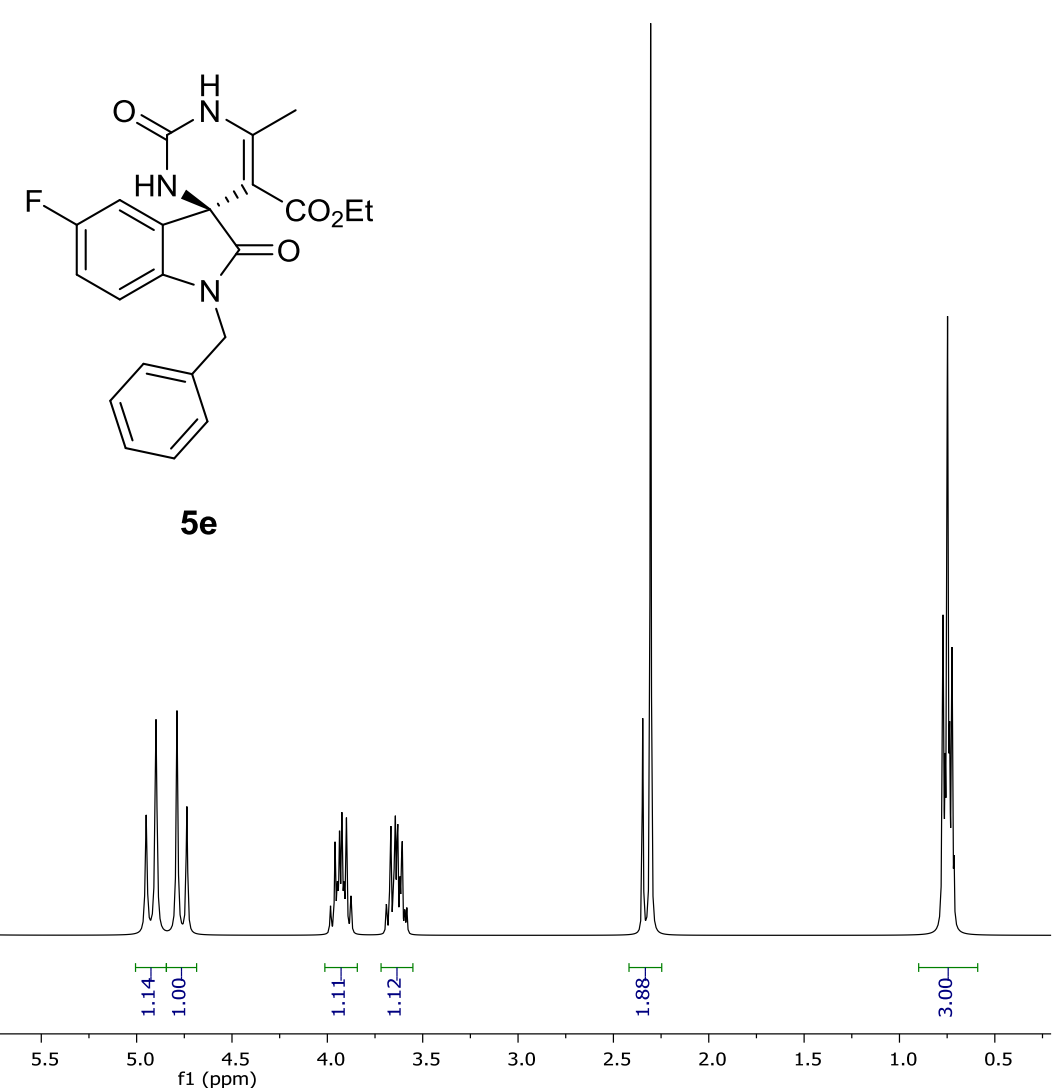

Compound 5e: ${ }^{13} \mathrm{C}$ NMR (75 MHz, $\mathrm{CDCl}_{3}$ )

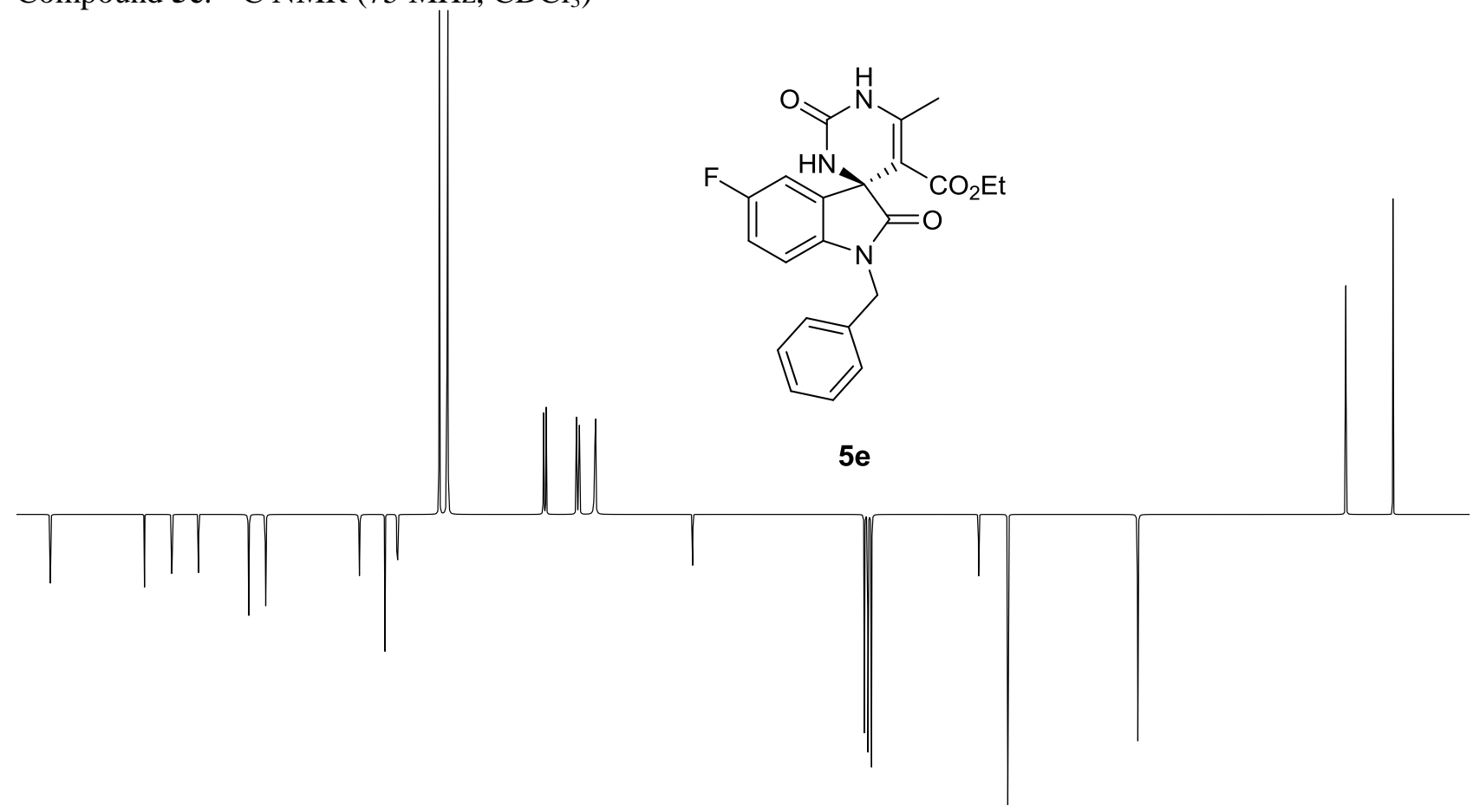


Compound 5f: ${ }^{1} \mathrm{H}$ NMR $\left(300 \mathrm{MHz}, \mathrm{CDCl}_{3}\right)$
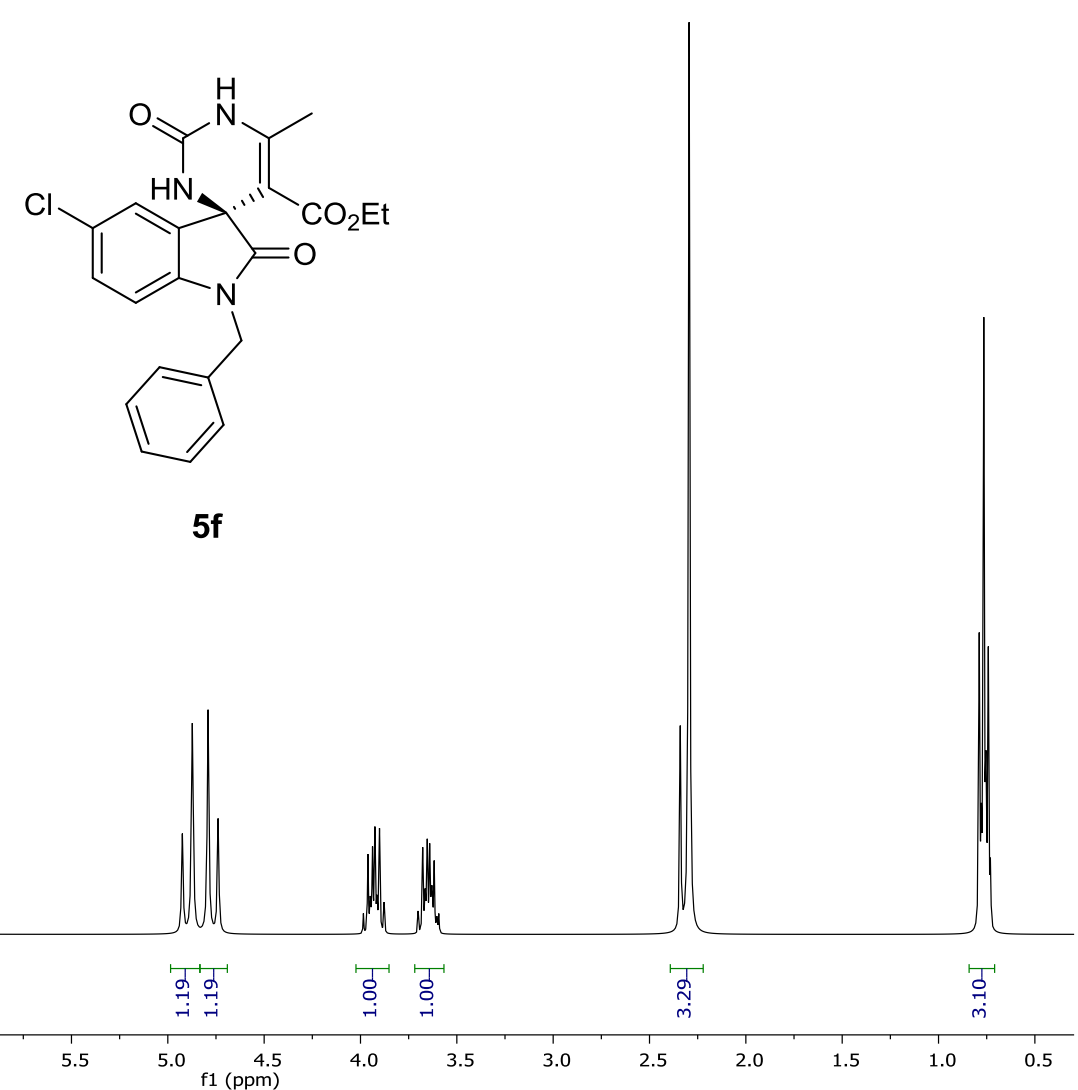

Compound 5f: ${ }^{13} \mathrm{C}$ NMR $\left(75 \mathrm{MHz}, \mathrm{CDCl}_{3}\right)$

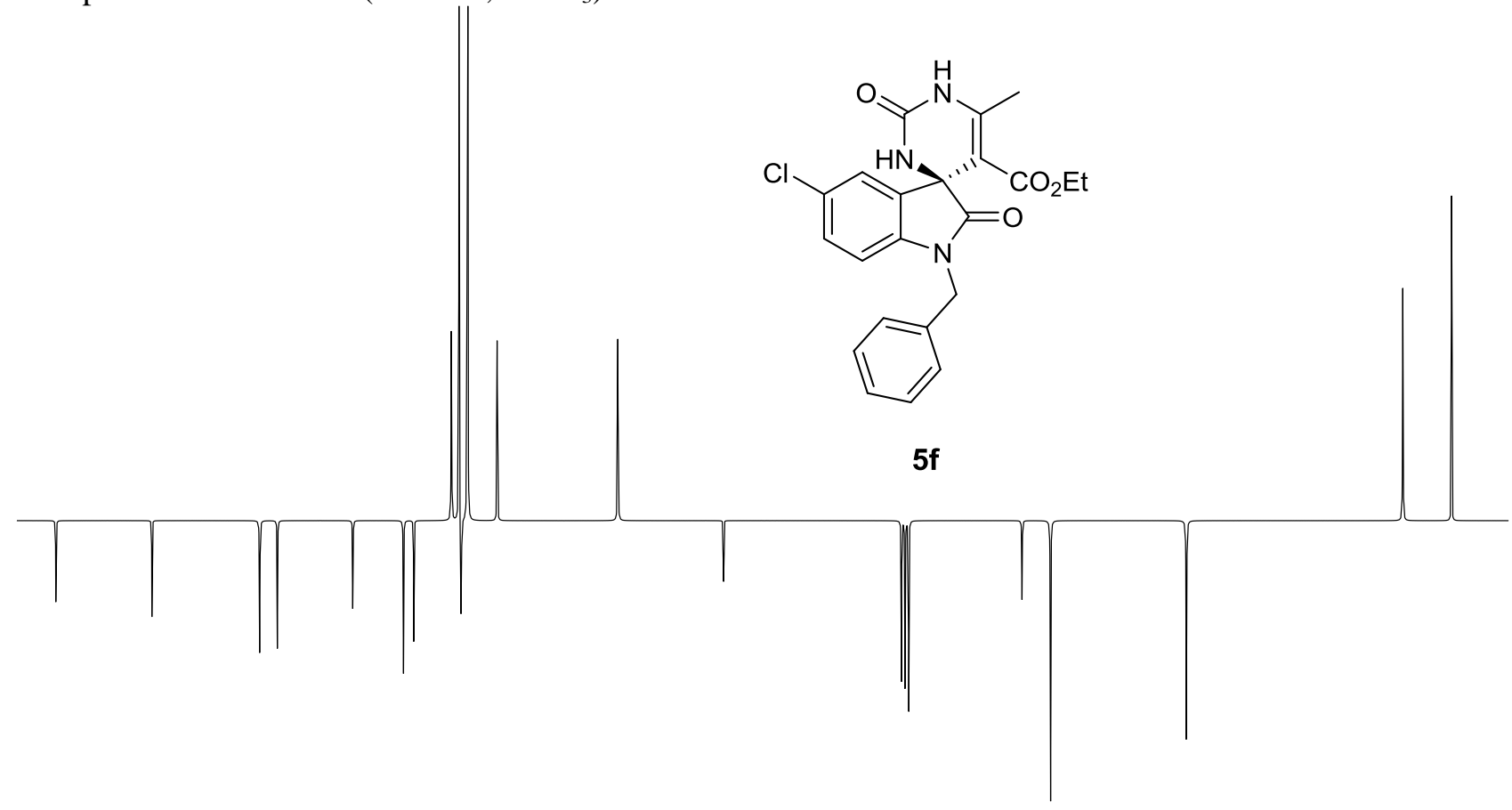

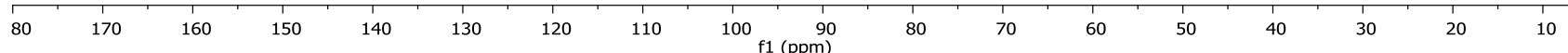


Compound 5g: ${ }^{1} \mathrm{H}$ NMR (300 MHz, $\mathrm{CDCl}_{3}$ )

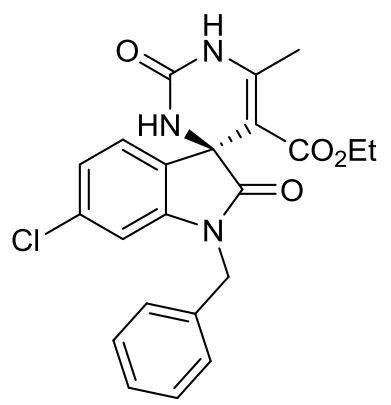

$5 g$

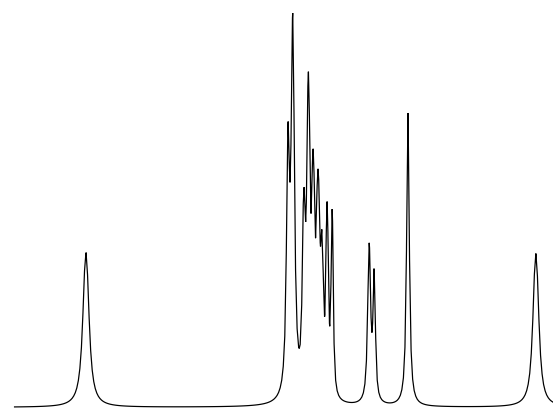$$
\text { g }
$$

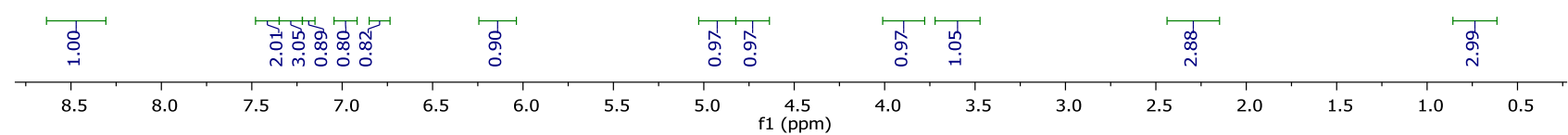

Compound 5g: ${ }^{13} \mathrm{C}$ NMR (75 MHz, $\mathrm{CDCl}_{3}$ )

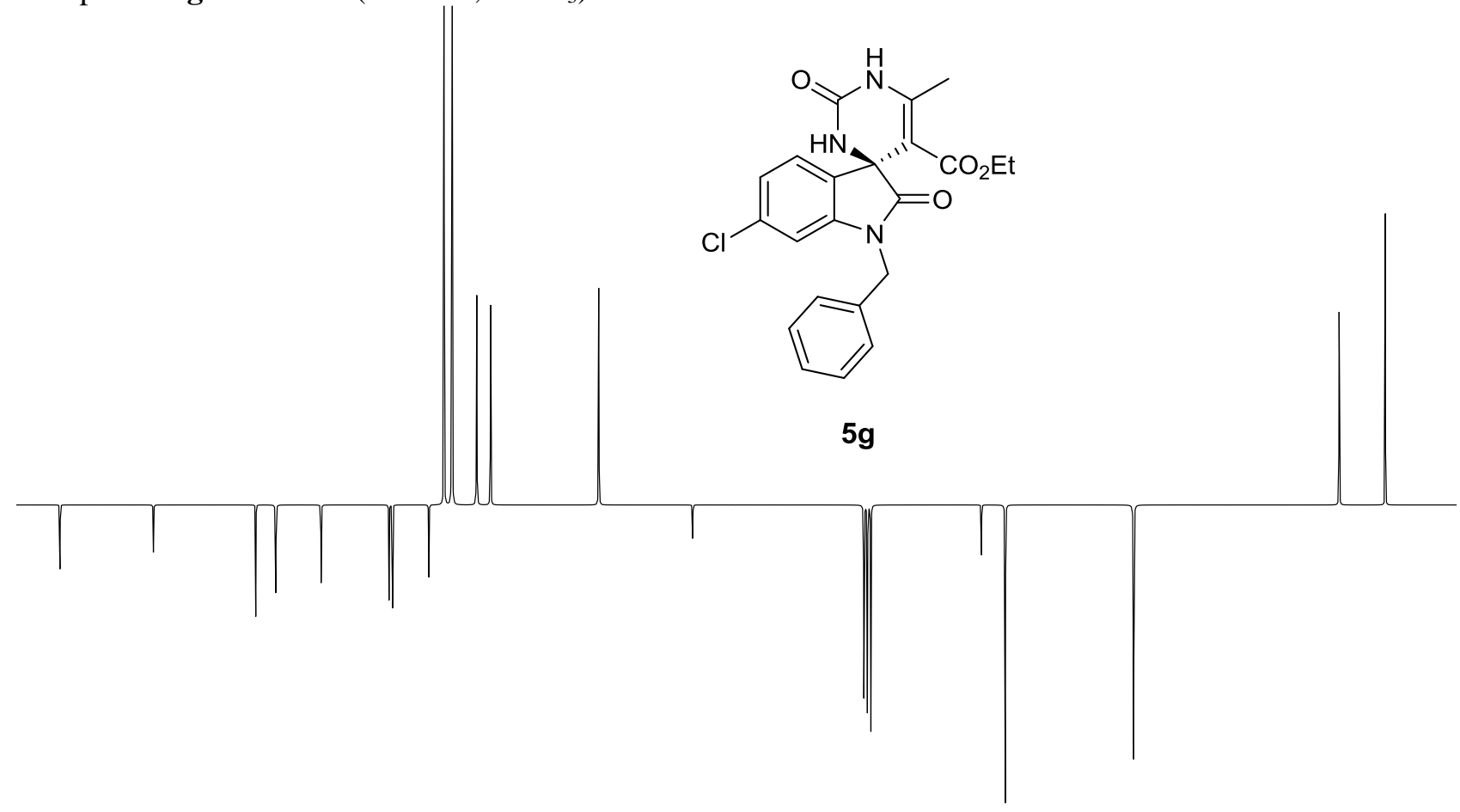


Compound 5h: ${ }^{1} \mathrm{H}$ NMR (300 MHz, $\mathrm{CDCl}_{3}$ )

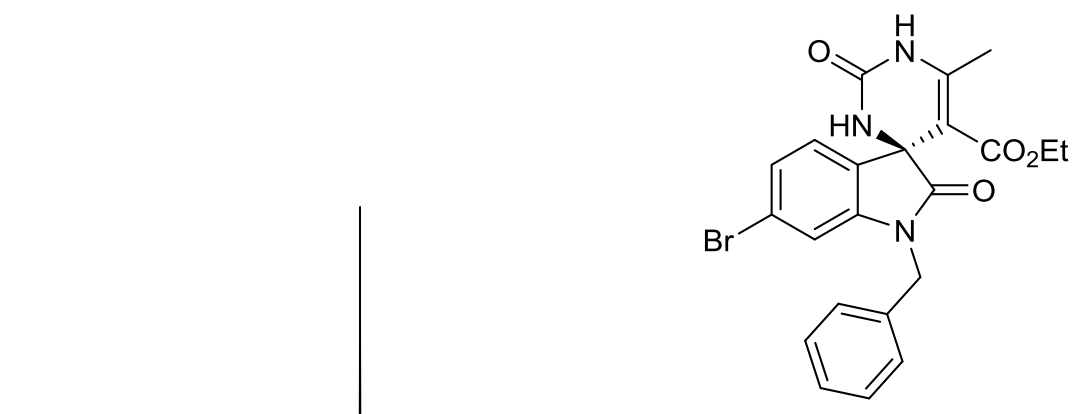

$5 \mathrm{~h}$

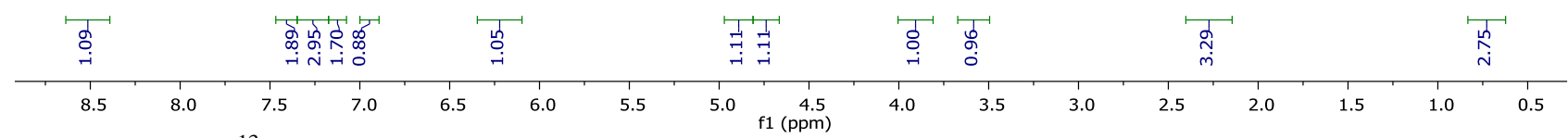

Compound 5h: ${ }^{13} \mathrm{C}$ NMR (75 MHz, $\mathrm{CDCl}_{3}$ )

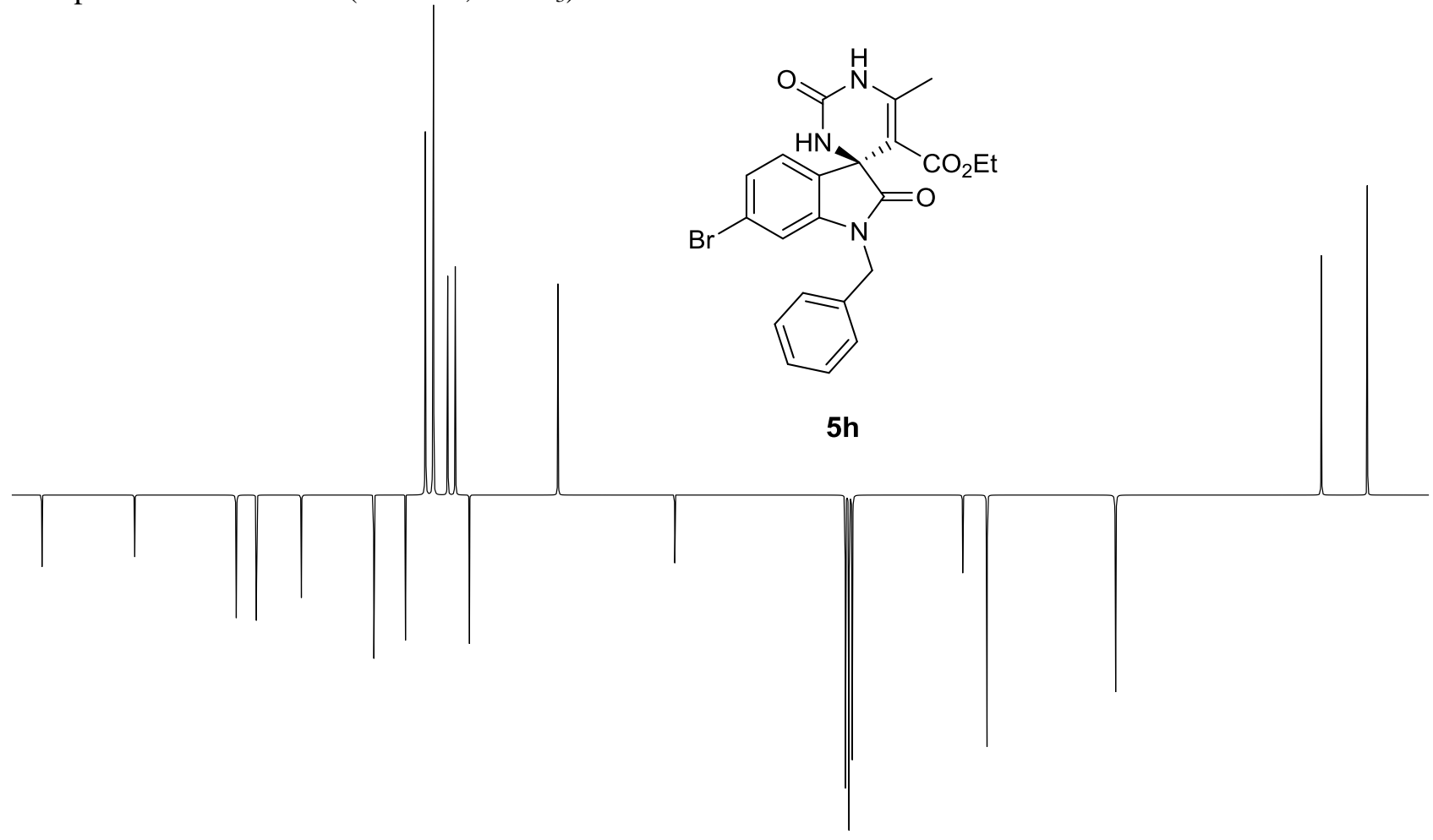

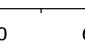

$60 \quad 50$


Compound 5i: ${ }^{1} \mathrm{H}$ NMR (300 MHz, $\mathrm{CDCl}_{3}$ )

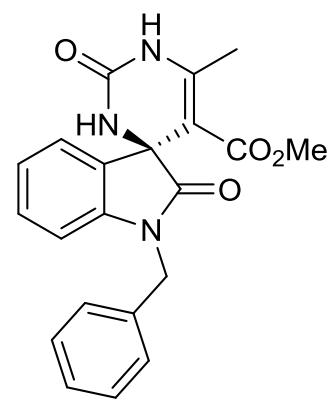

$5 \mathbf{i}$

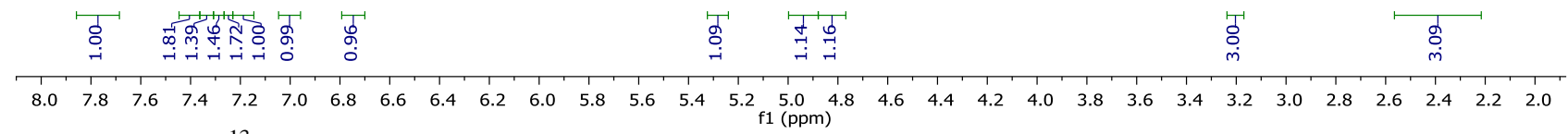

Compound 5i: ${ }^{13} \mathrm{C}$ NMR (75 MHz, $\mathrm{CDCl}_{3}$ )

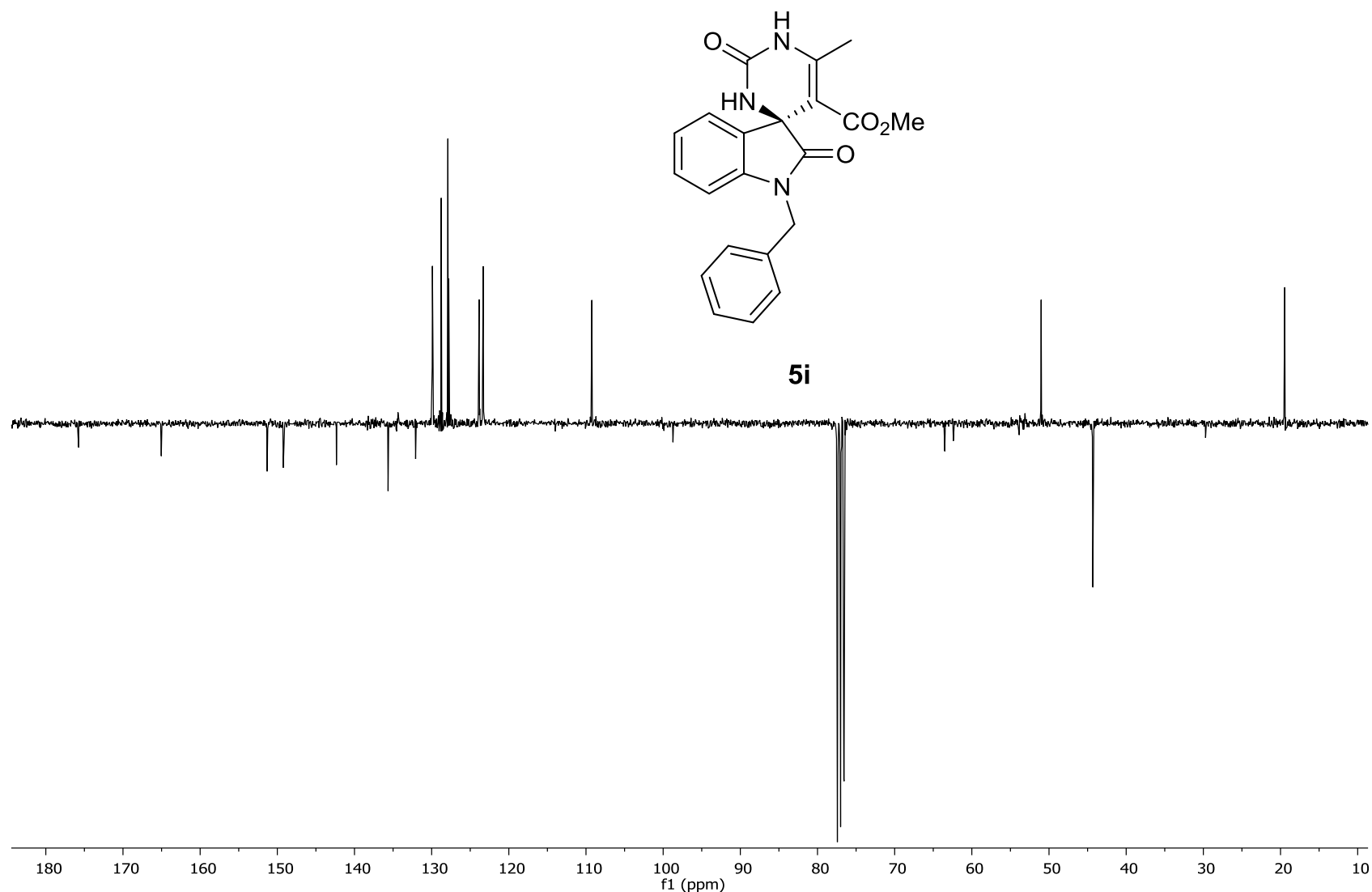


Compound 5j: ${ }^{1} \mathrm{H}$ NMR $\left(300 \mathrm{MHz}, \mathrm{CDCl}_{3}\right)$

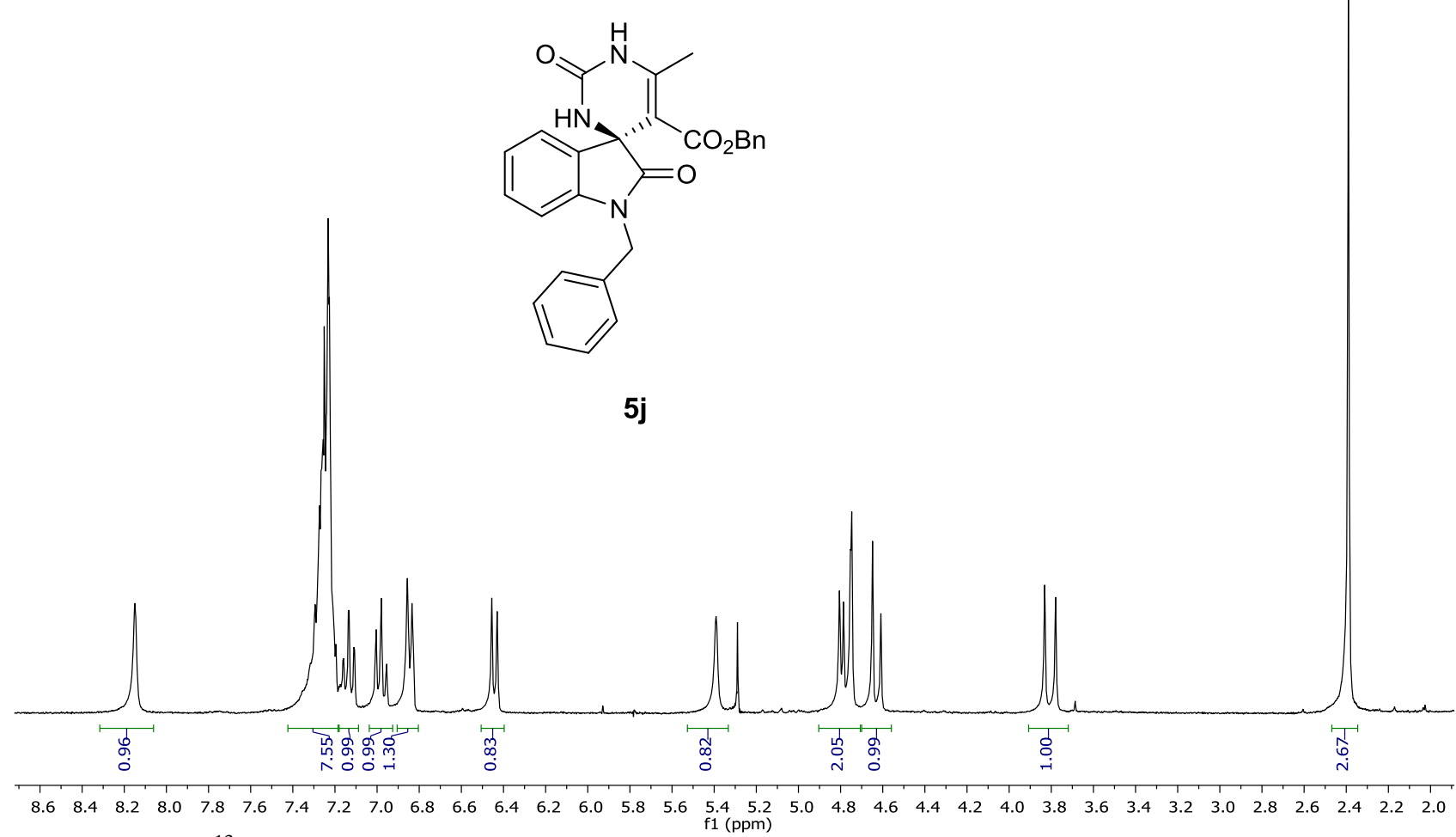

Compound 5j: ${ }^{13} \mathrm{C}$ NMR $\left(75 \mathrm{MHz}, \mathrm{CDCl}_{3}\right)$

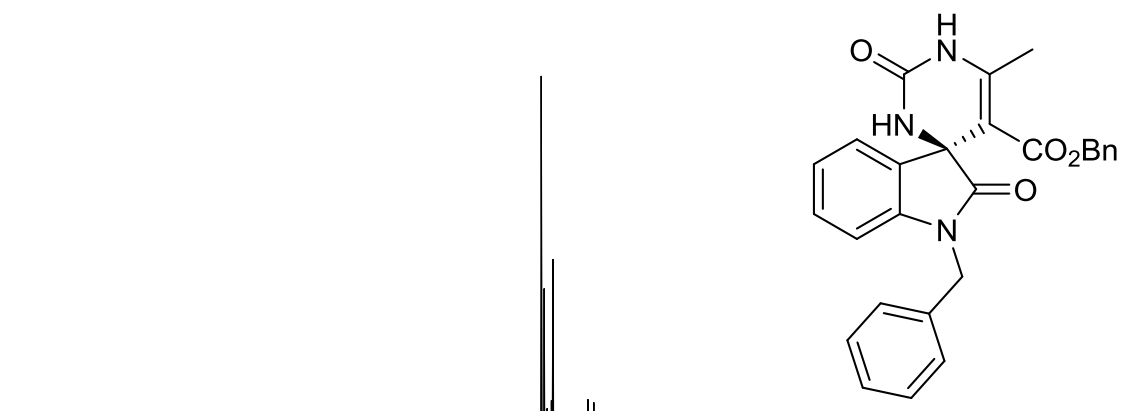

5j

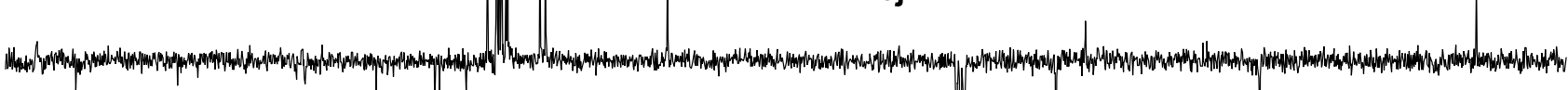


Compound 6: ${ }^{1} \mathrm{H}$ NMR (300 MHz, $\mathrm{CDCl}_{3}$ )

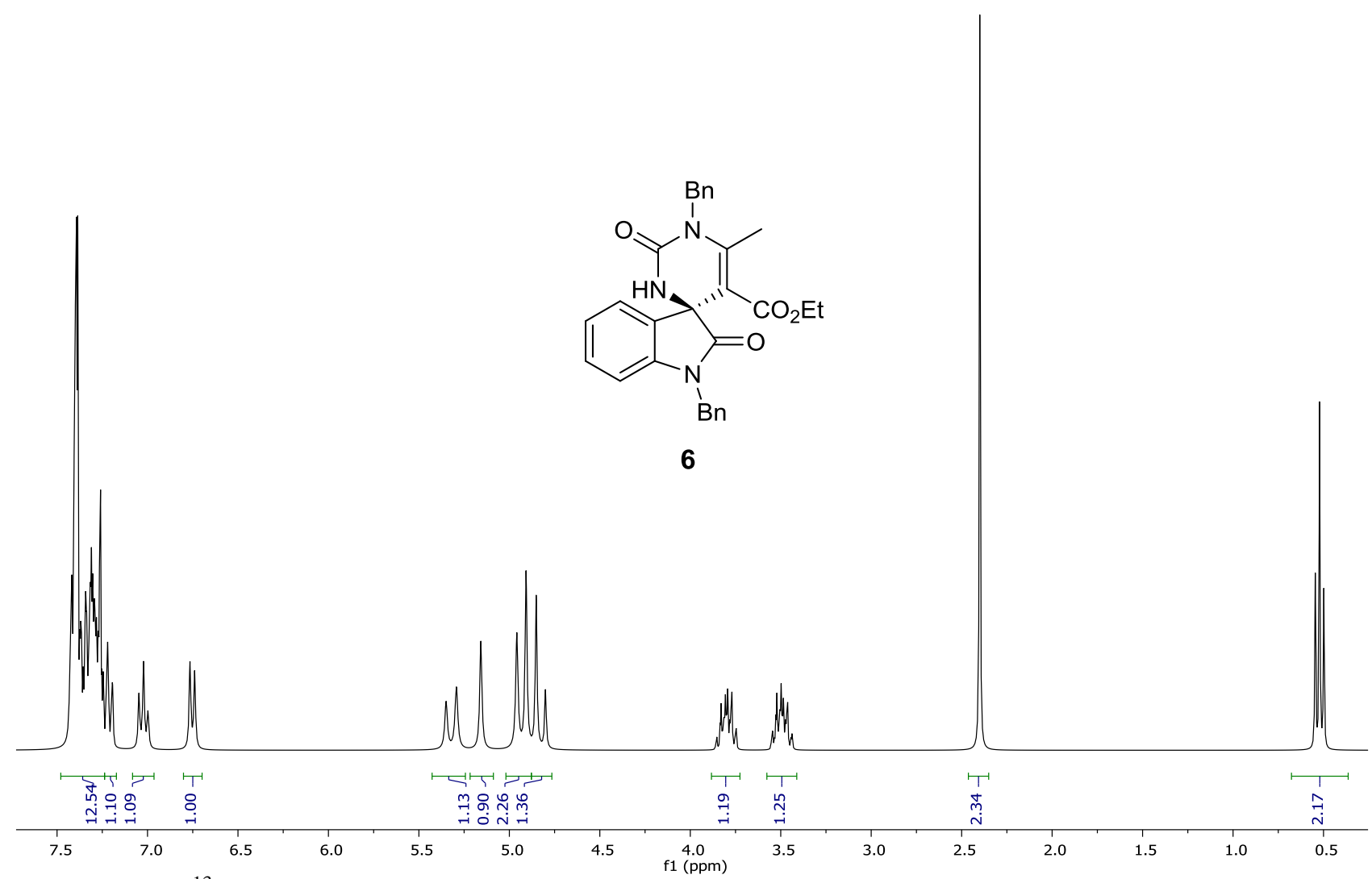

Compound 6: ${ }^{13} \mathrm{C}$ NMR $\left(75 \mathrm{MHz}, \mathrm{CDCl}_{3}\right)$

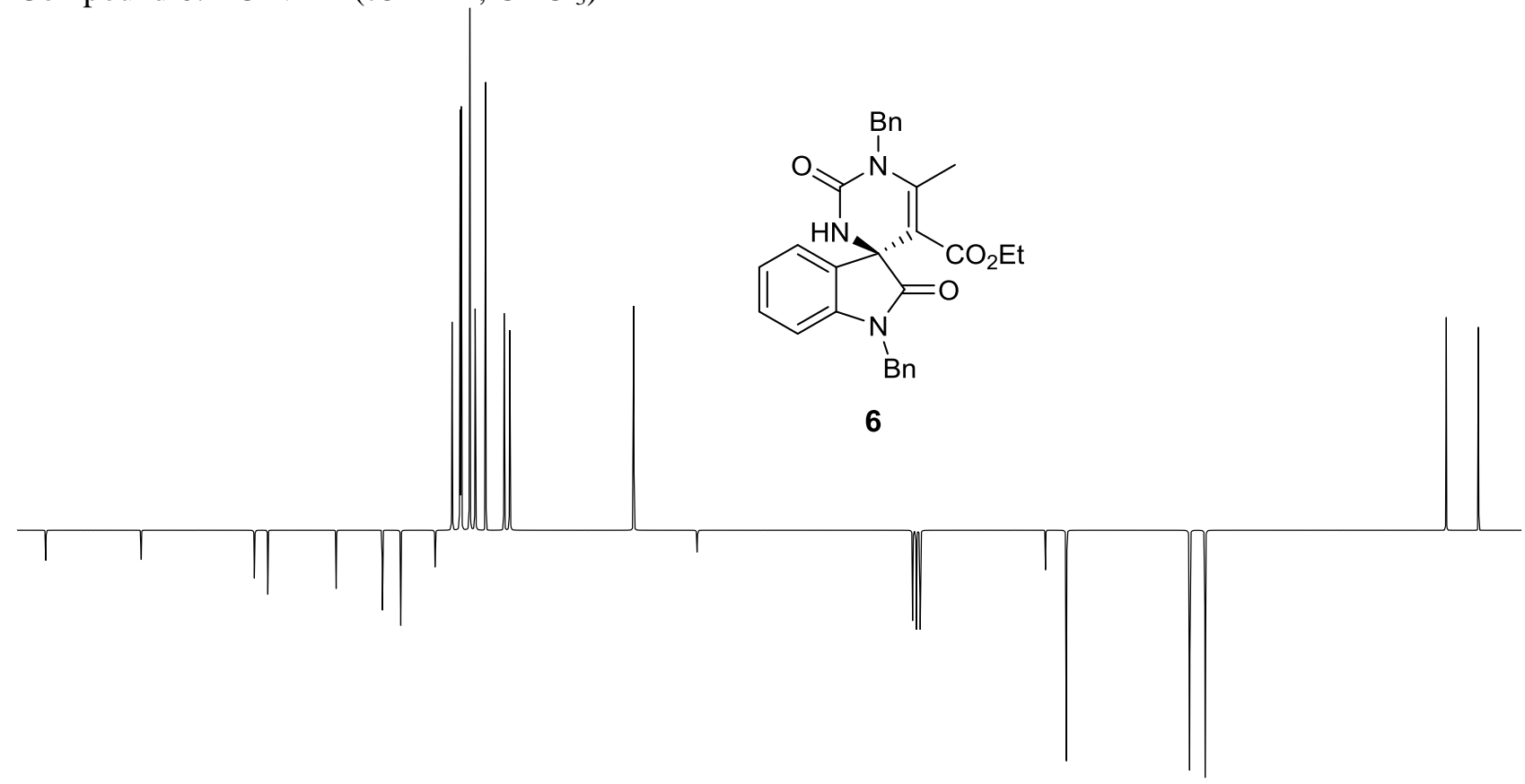

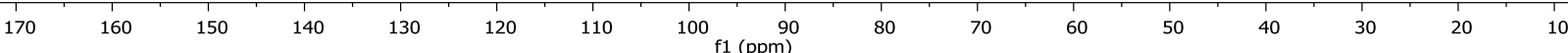


Compound 7: ${ }^{1} \mathrm{H}$ NMR (300 MHz, DMSO- $d_{6}$ )

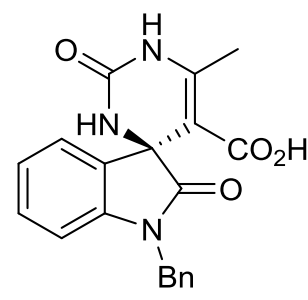

7

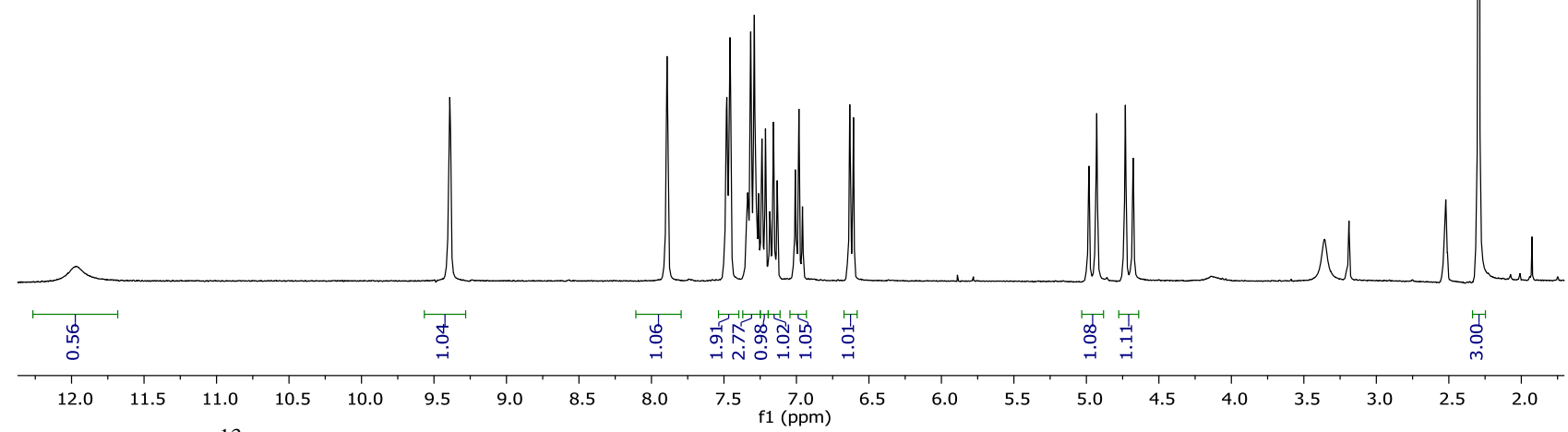

Compound 7: ${ }^{13} \mathrm{C}$ NMR (75 MHz, DMSO- $d_{6}$ )
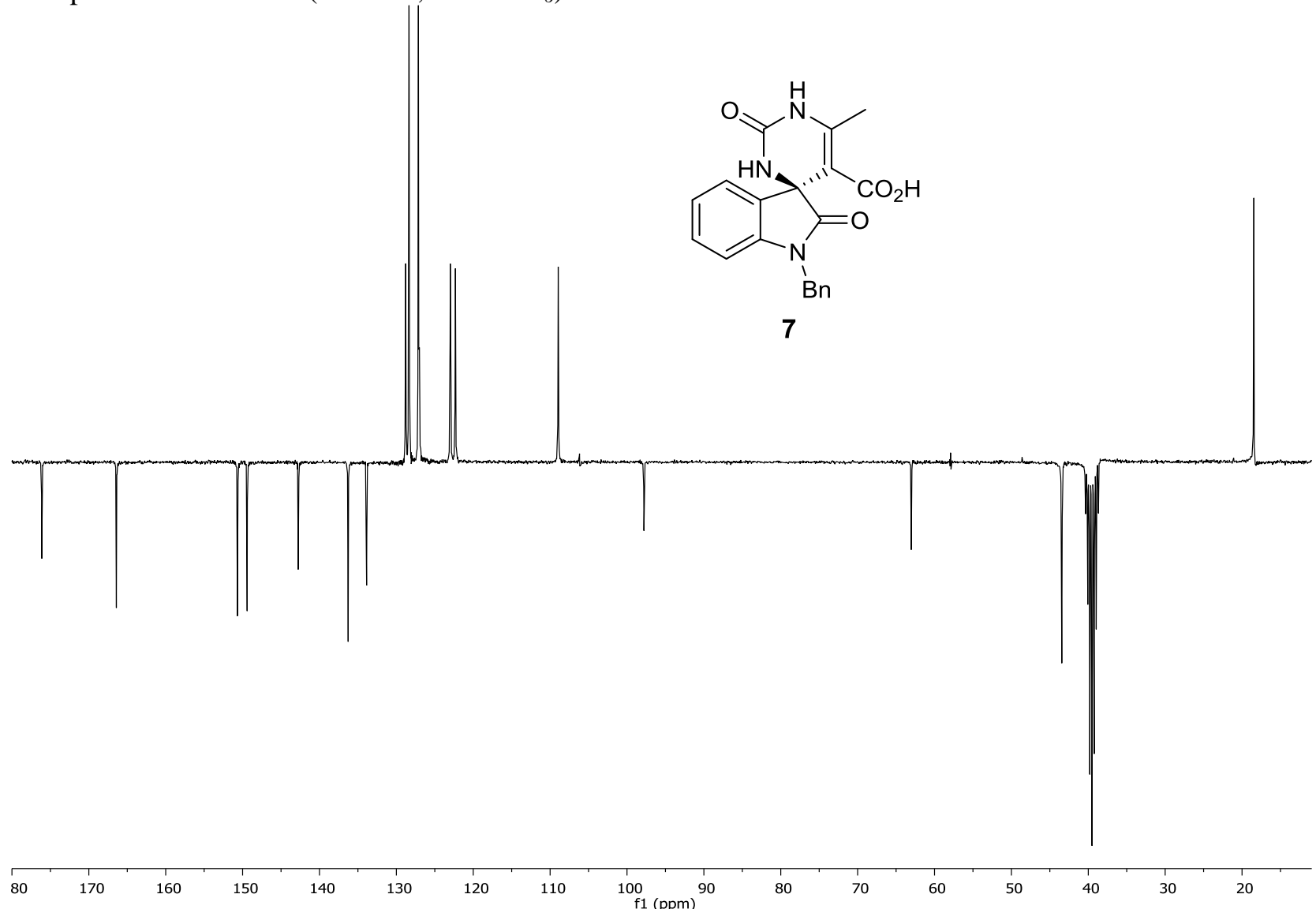
Compound 8: ${ }^{1} \mathrm{H}$ NMR (400 MHz, $\mathrm{CDCl}_{3}$ )

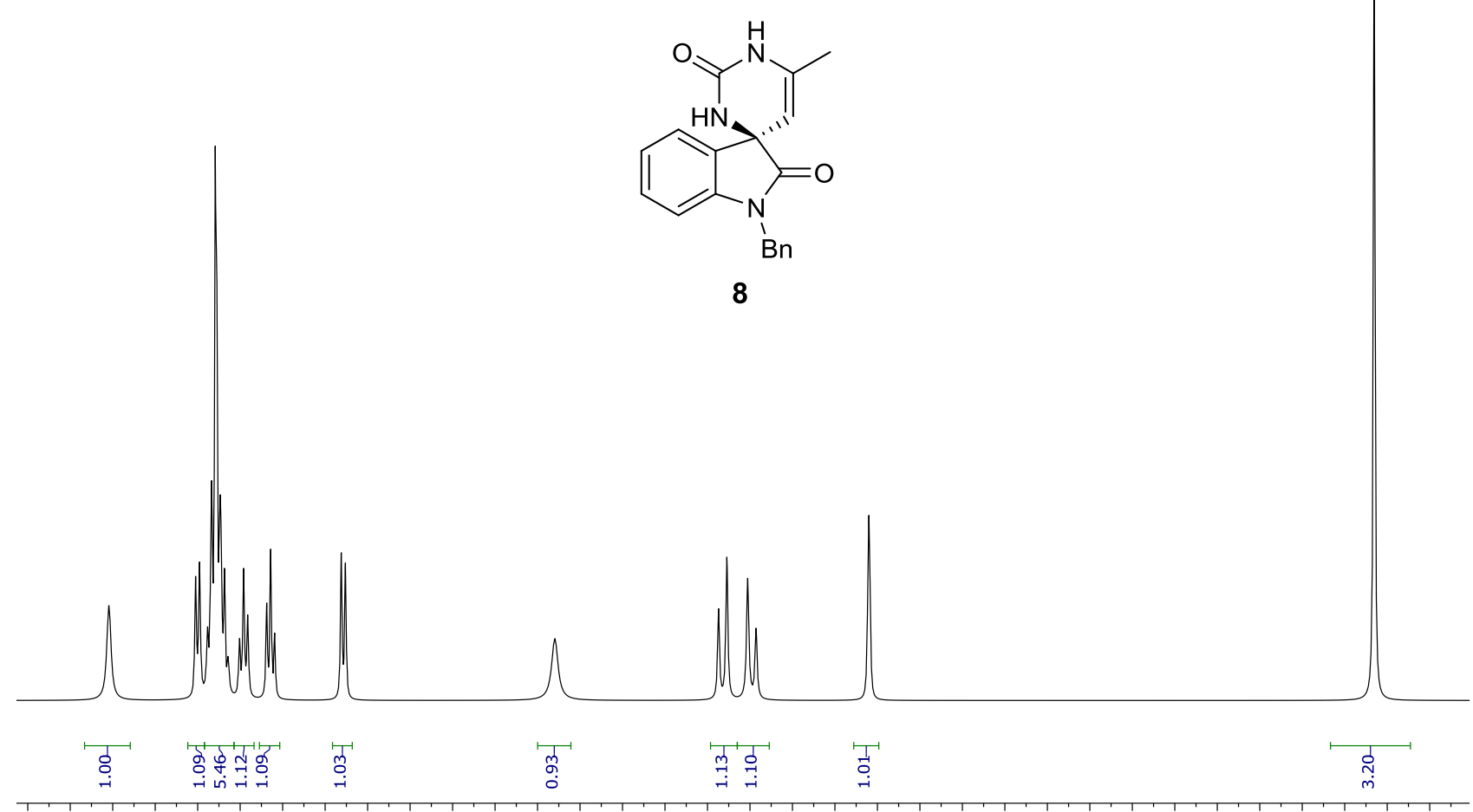

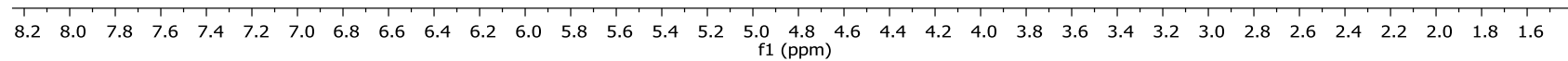
Compound 8: ${ }^{13} \mathrm{C}$ NMR (100 MHz, $\left.\mathrm{CDCl}_{3}\right)$

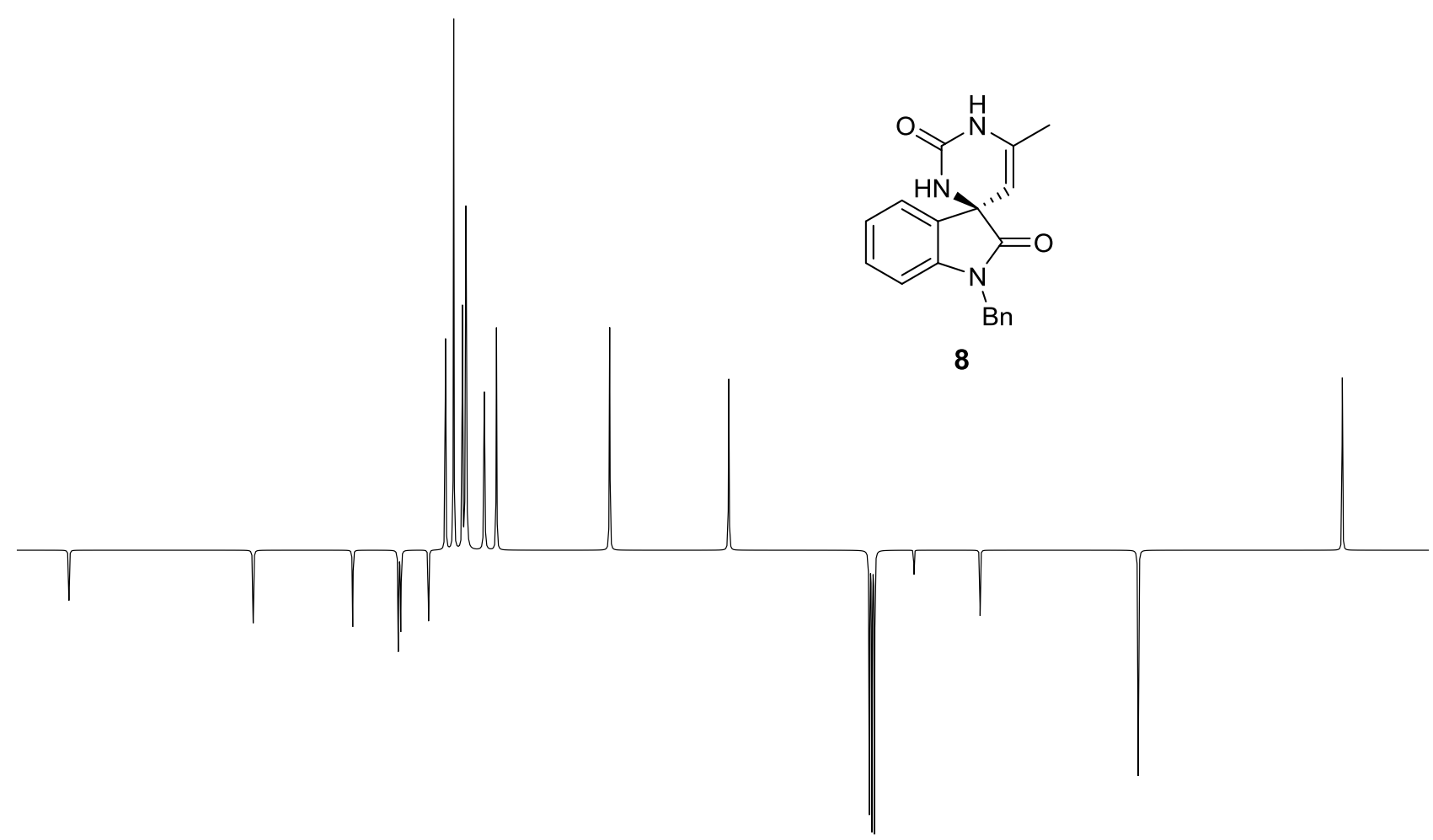

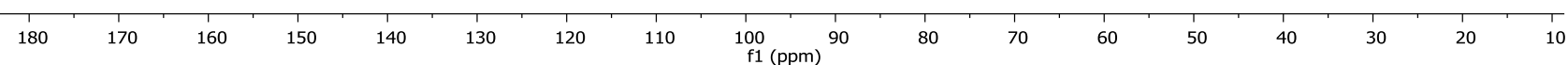


Compound 9a: ${ }^{1} \mathrm{H}$ NMR (400 MHz, DMSO- $d_{6}$ )

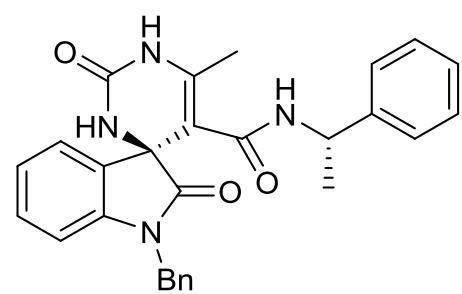

9a $(S, S)$

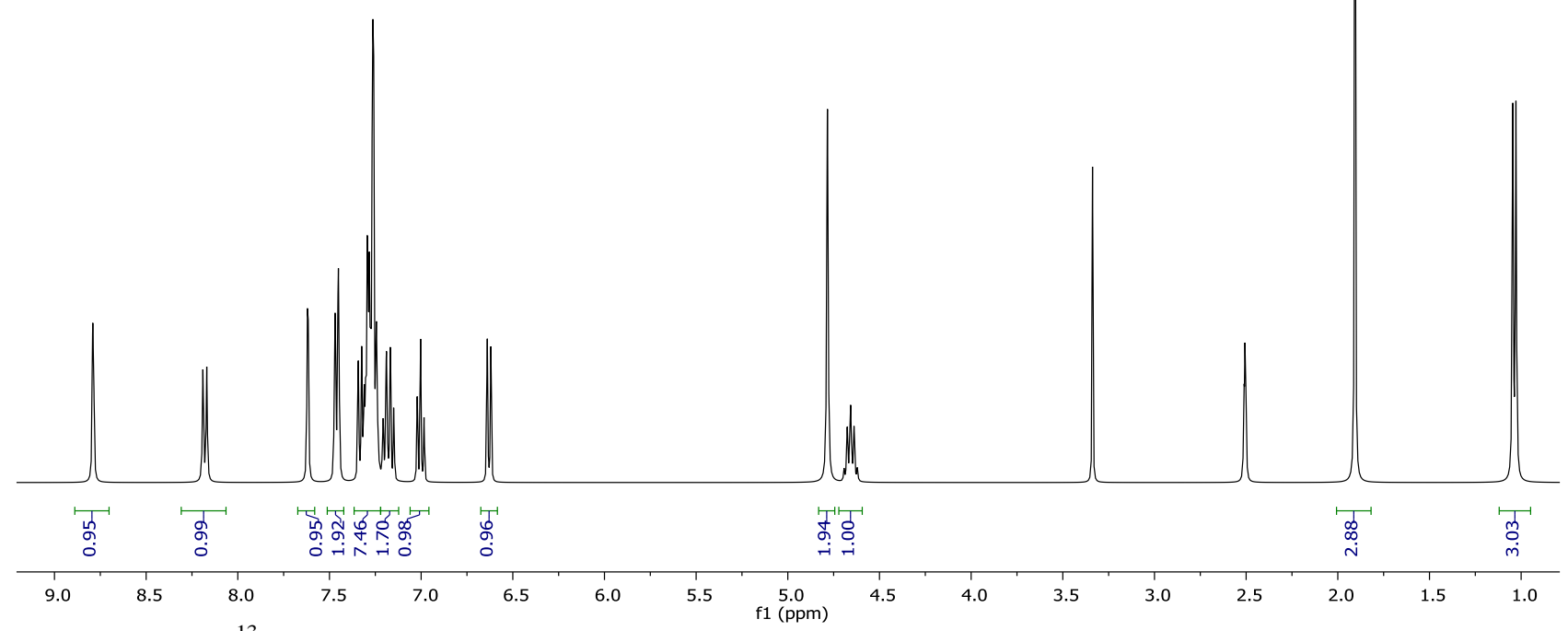

Compound 9a: ${ }^{13} \mathrm{C}$ NMR (100 MHz, DMSO- $d_{6}$ )

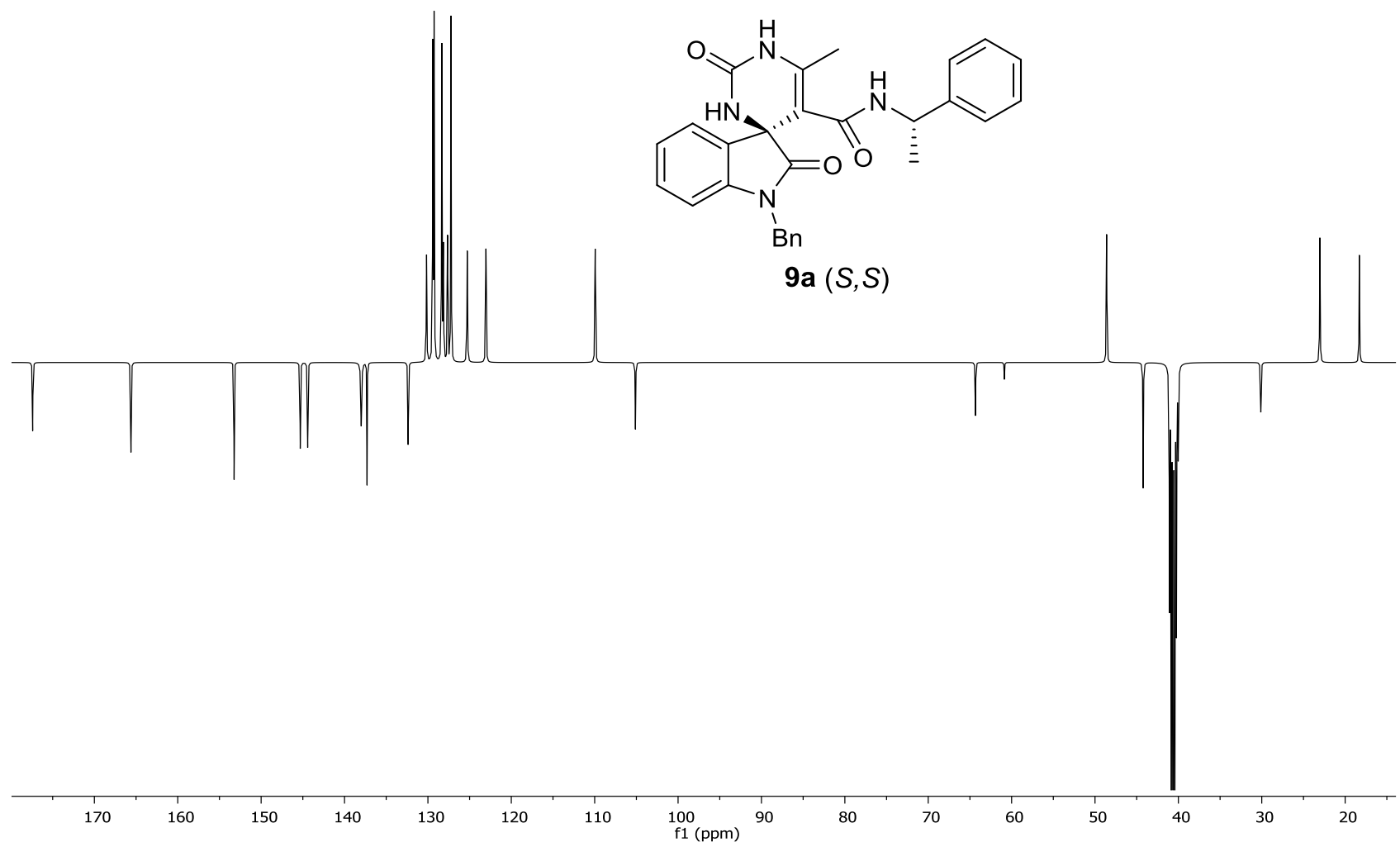


Compound 9b: ${ }^{1} \mathrm{H}$ NMR (400 MHz, DMSO- $d_{6}$ )
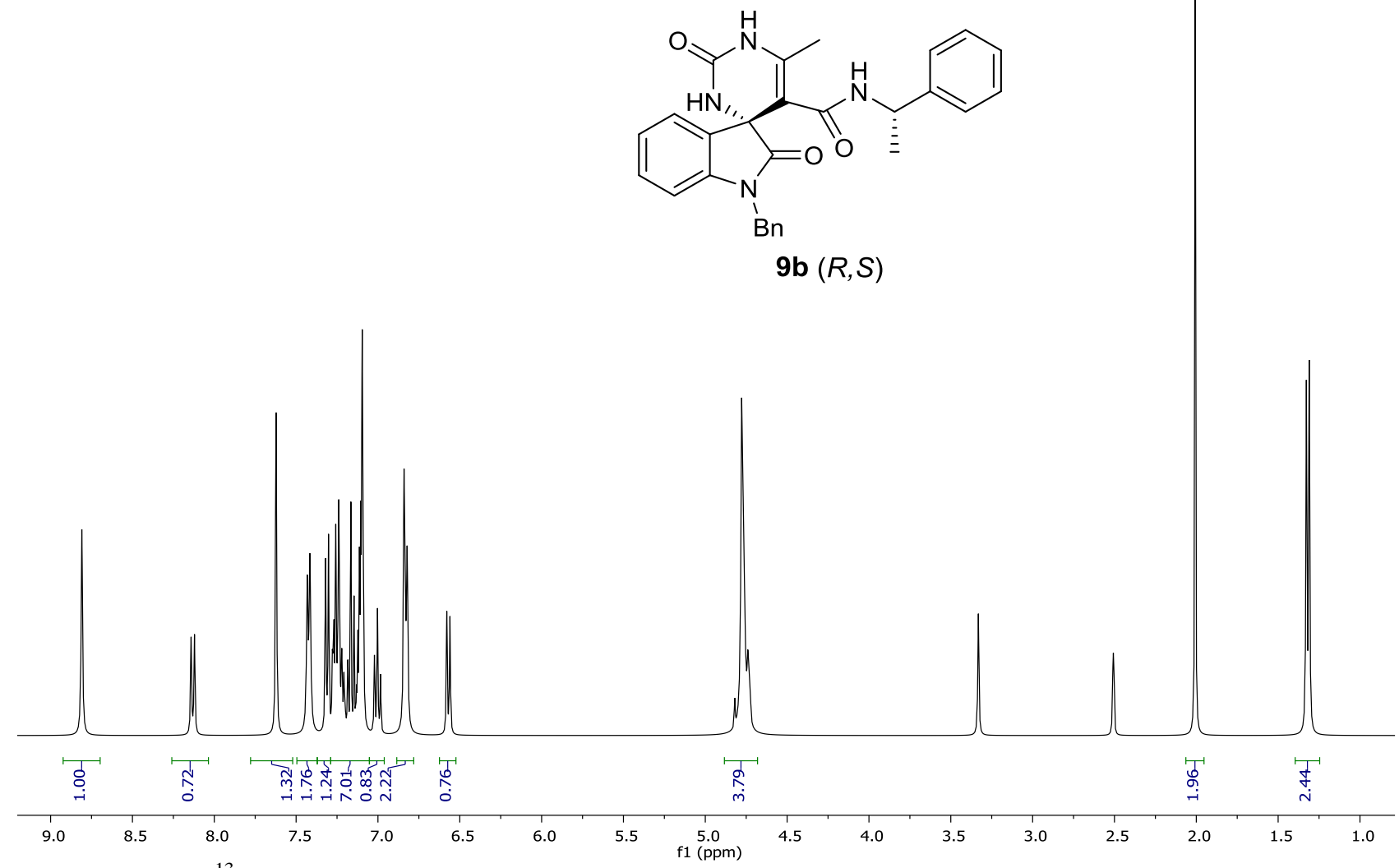

Compound 9b: ${ }^{13} \mathrm{C}$ NMR (100 MHz, DMSO- $d_{6}$ )

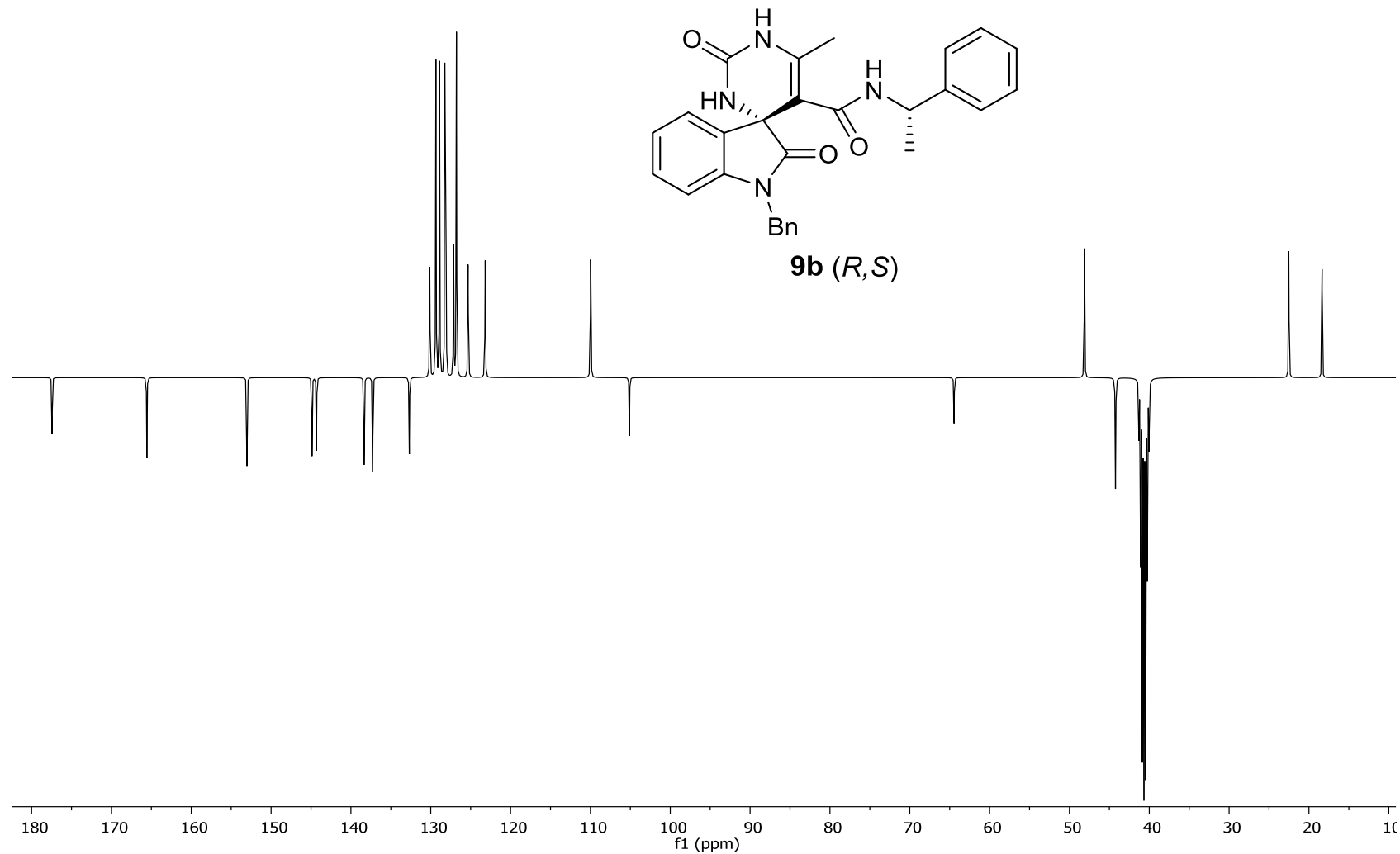




\section{HPLC chromatograms}

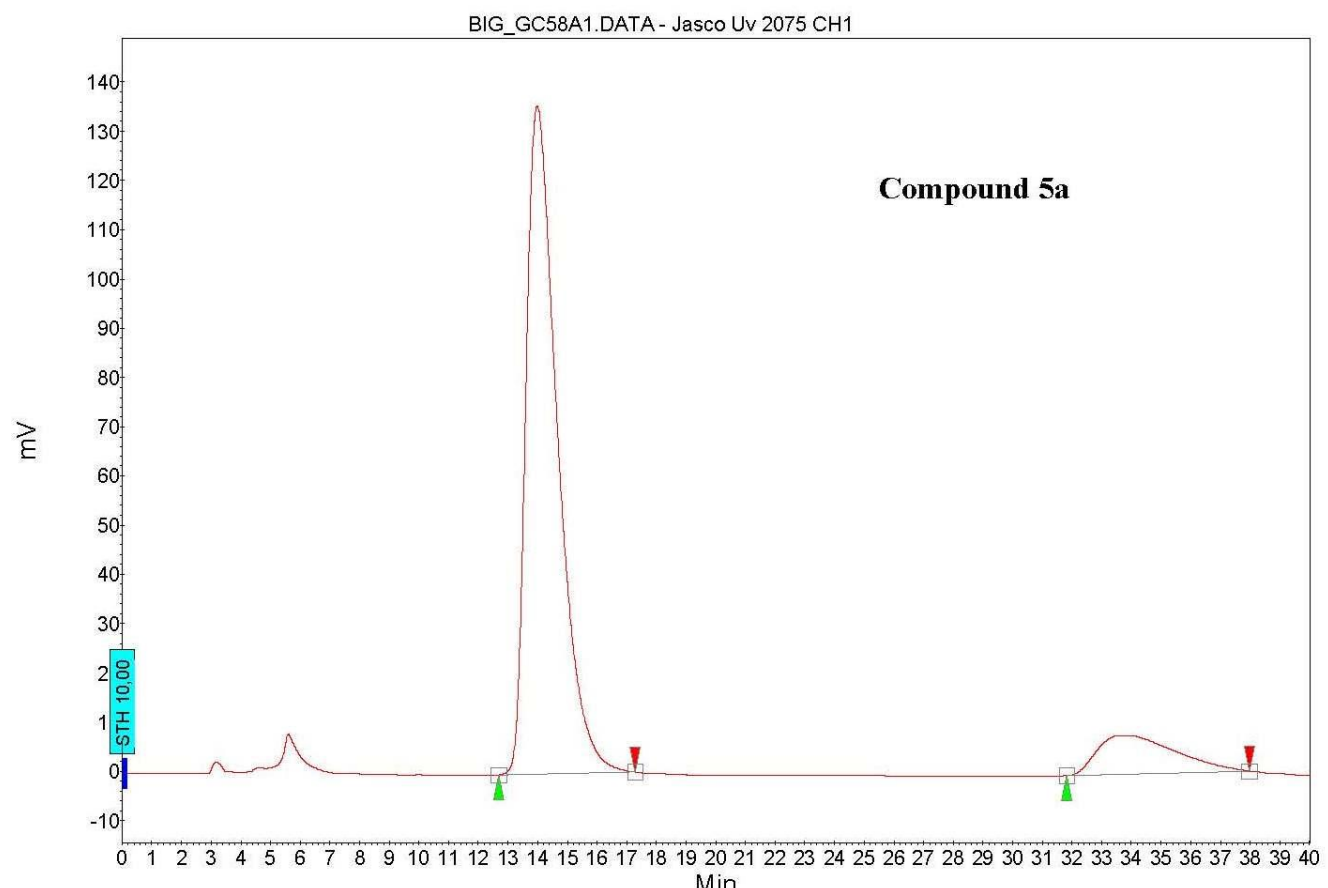

Min

$1 \mathrm{mg}$ in $2 \mathrm{~mL} \mathrm{iPrOH}$

\begin{tabular}{|c|l|r|r|r|r|r|r|r|r|}
\hline Index & Name & Start & Time & End & Ret. time Offset & Quantity & Height & Area & Area \% \\
\hline \hline & & {$[\mathrm{Min}]$} & {$[\mathrm{Min}]$} & {$[\mathrm{Min}]$} & {$[\mathrm{Min}]$} & {$[\%$ Area] } & {$[\mathrm{mV}]$} & {$[\mathrm{mV} . \mathrm{Min}]$} & {$[\%]$} \\
\hline 1 & UNKNOWN & 12,683 & 13,983 & 17,283 & 0,000 & 89,94 & 135,7 & 171,7 & 89,942 \\
\hline 2 & UNKNOWN & 31,833 & 33,683 & 37,967 & 0,000 & 10,06 & 7,9 & 19,2 & 10,058 \\
\hline & & & & & & & & & \\
\hline Total & & & & & & 100,00 & 143,6 & 190,9 & 100,000 \\
\hline
\end{tabular}

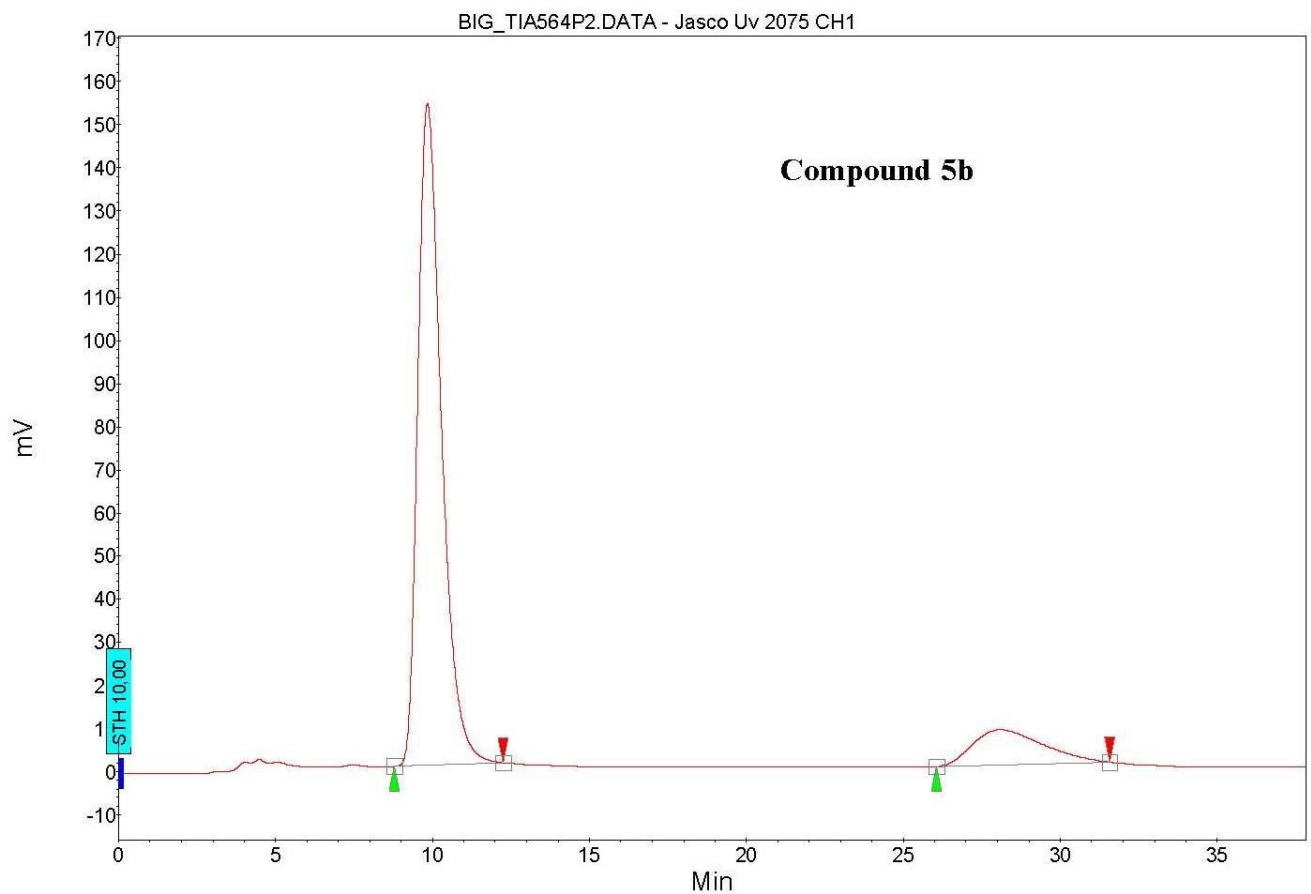

$1 \mathrm{mg}$ in $2 \mathrm{~mL}$ iPrOH

\begin{tabular}{|c|l|r|r|r|r|r|r|r|r|}
\hline Index & Name & Start & Time & End & Ret. time Offset & Quantity & Height & Area & Area \% \\
\hline \hline & & {$[\mathrm{Min}]$} & {$[\mathrm{Min}]$} & {$[\mathrm{Min}]$} & {$[\mathrm{Min}]$} & {$[\%$ Area $]$} & {$[\mathrm{mV}]$} & {$[\mathrm{mV} . \mathrm{Min}]$} & {$[\%]$} \\
\hline 1 & UNKNOWN & 8,783 & 9,842 & 12,250 & 0,000 & 87,45 & 153,4 & 135,9 & 87,452 \\
\hline 2 & UNKNOWN & 26,050 & 28,058 & 31,567 & 0,000 & 12,55 & 8,2 & 19,5 & 12,548 \\
\hline & & & & & & & & & \\
\hline Total & & & & & & 100,00 & 161,6 & 155,4 & 100,000 \\
\hline
\end{tabular}




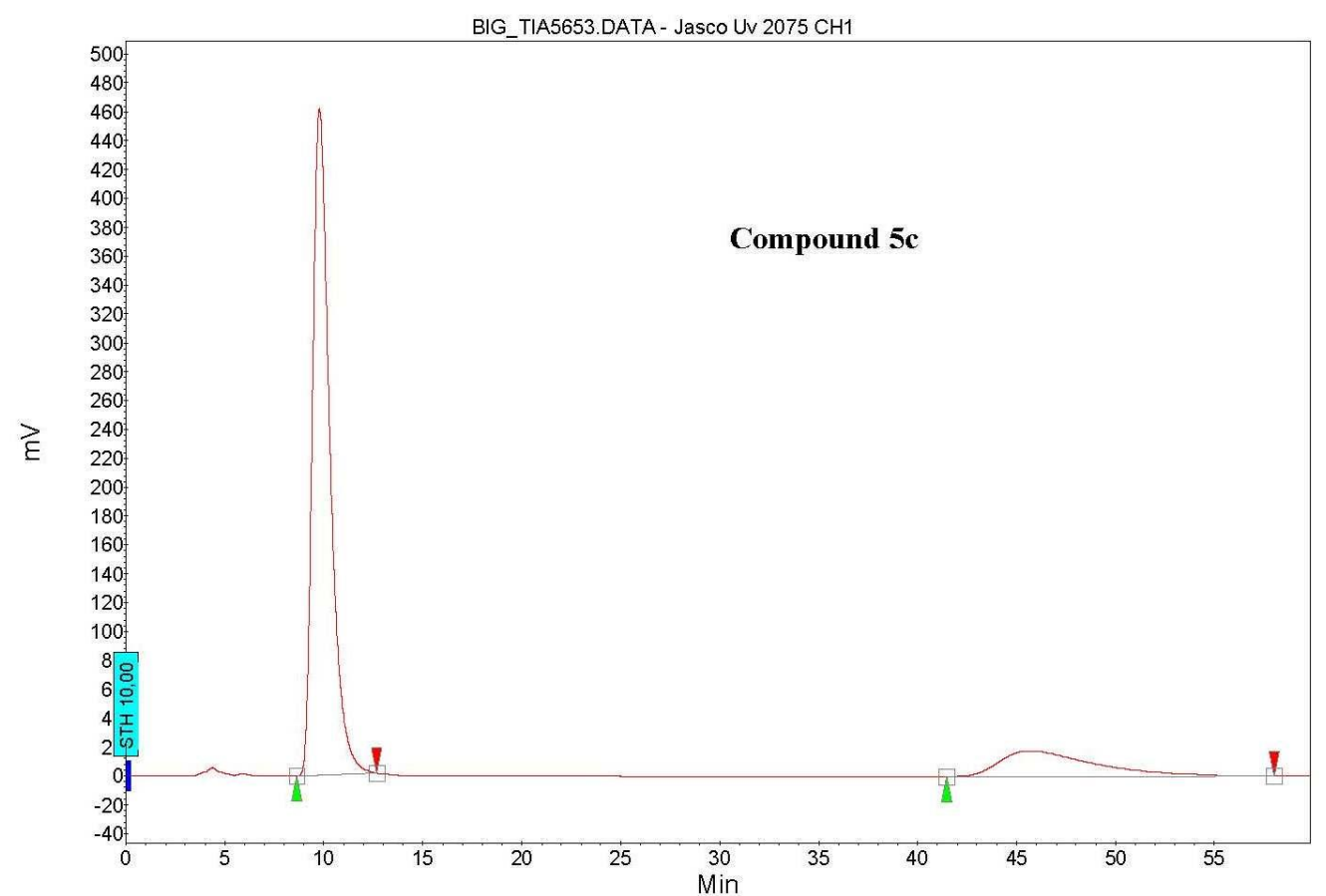

$1 \mathrm{mg}$ in $2 \mathrm{~mL}$ iPrOH

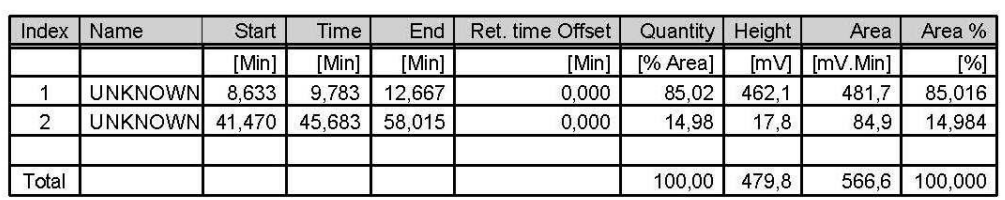

BIG_TIA563P2.DATA - Jasco Uv $2075 \mathrm{CH}$

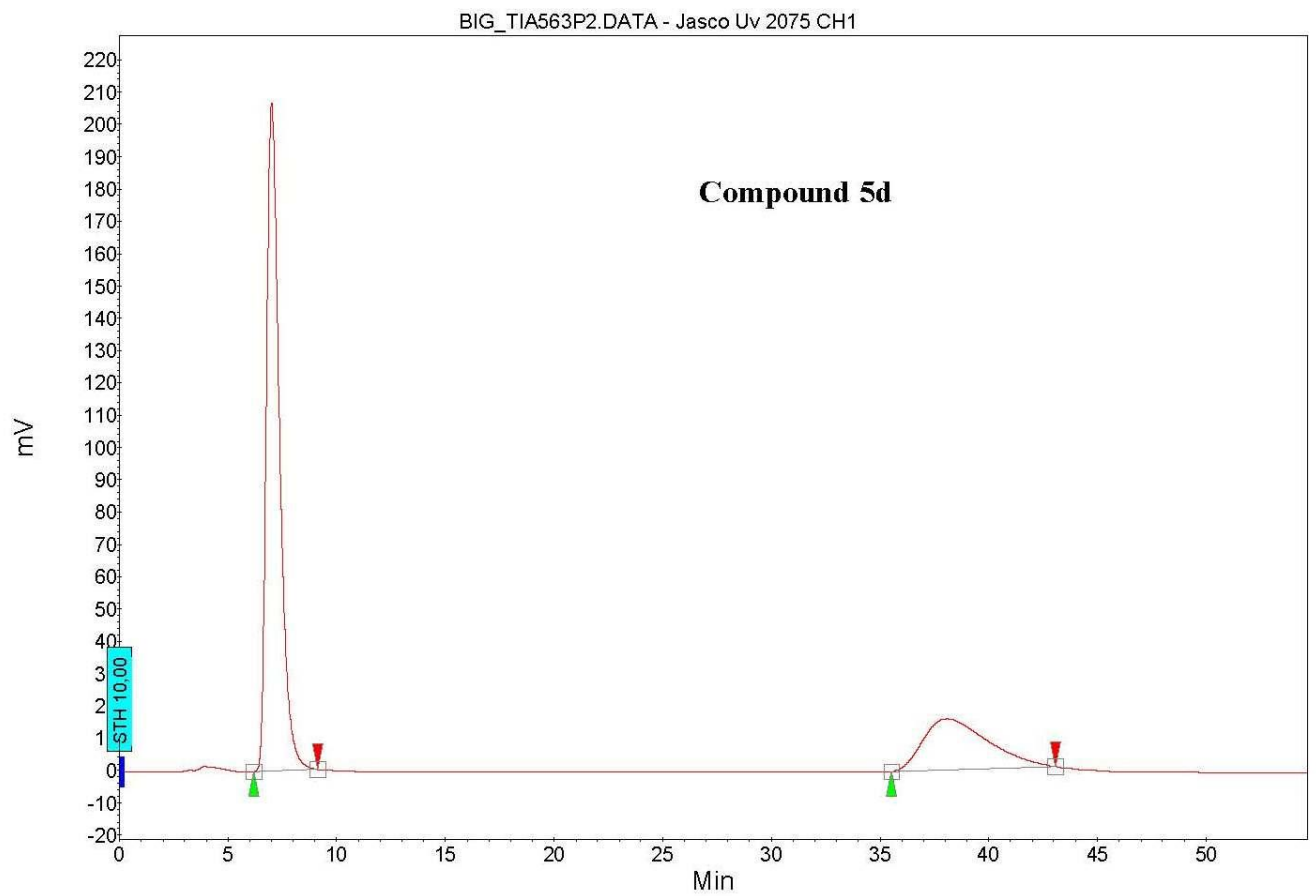

$1 \mathrm{mg} 2 \mathrm{ml} \mathrm{iPrOH}$

\begin{tabular}{|c|l|r|r|r|r|r|r|r|r|}
\hline Index & Name & Start & Time & End & Ret. time Offset & Quantity & Height & Area & Area \% \\
\hline \hline & & {$[\mathrm{Min}]$} & {$[\mathrm{Min}]$} & {$[\mathrm{Min}]$} & {$[\mathrm{Min}]$} & {$[\%$ Area] } & {$[\mathrm{mV}]$} & {$[\mathrm{mV} . \mathrm{Min}]$} & {$[\%]$} \\
\hline 1 & UNKNOWN & 6,183 & 7,008 & 9,117 & 0,000 & 75,04 & 206,8 & 149,7 & 75,038 \\
\hline 2 & UNKNOWN & 35,517 & 38,058 & 43,067 & 0,000 & 24,96 & 15,9 & 49,8 & 24,962 \\
\hline & & & & & & & & & \\
\hline Total & & & & & & 100,00 & 222,7 & 199,5 & 100,000 \\
\hline
\end{tabular}




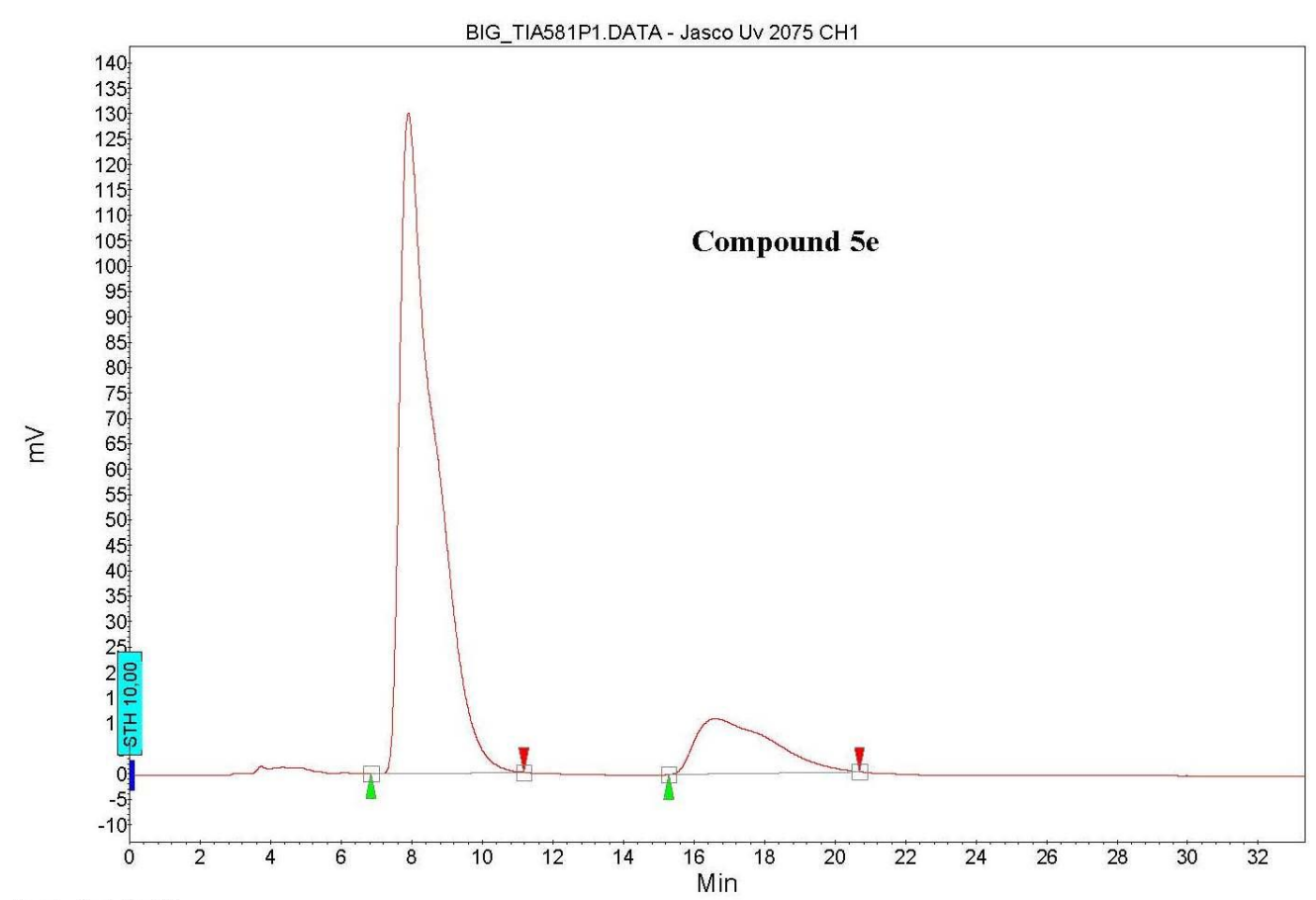

$1 \mathrm{mg}$ in $2 \mathrm{~mL} \mathrm{iPrOH}$

\begin{tabular}{|c|l|r|r|r|r|r|r|r|r|}
\hline Index & Name & Start & Time & End & Ret. time Offset & Quantity & Height & Area & Area \% \\
\hline \hline & & {$[\mathrm{Min}]$} & {$[\mathrm{Min}]$} & {$[\mathrm{Min}]$} & {$[\mathrm{Min}]$} & {$[\%$ Area] } & {$[\mathrm{mV}]$} & {$[\mathrm{mV} . \mathrm{Min}]$} & {$[\%]$} \\
\hline 1 & UNKNOWN & 6,842 & 7,908 & 11,183 & 0,000 & 87,53 & 130,0 & 153,0 & 87,529 \\
\hline 2 & UNKNOWN & 15,283 & 16,617 & 20,683 & 0,000 & 12,47 & 10,9 & 21,8 & 12,471 \\
\hline & & & & & & & & & \\
\hline Total & & & & & & 100,00 & 140,9 & 174,8 & 100,000 \\
\hline
\end{tabular}

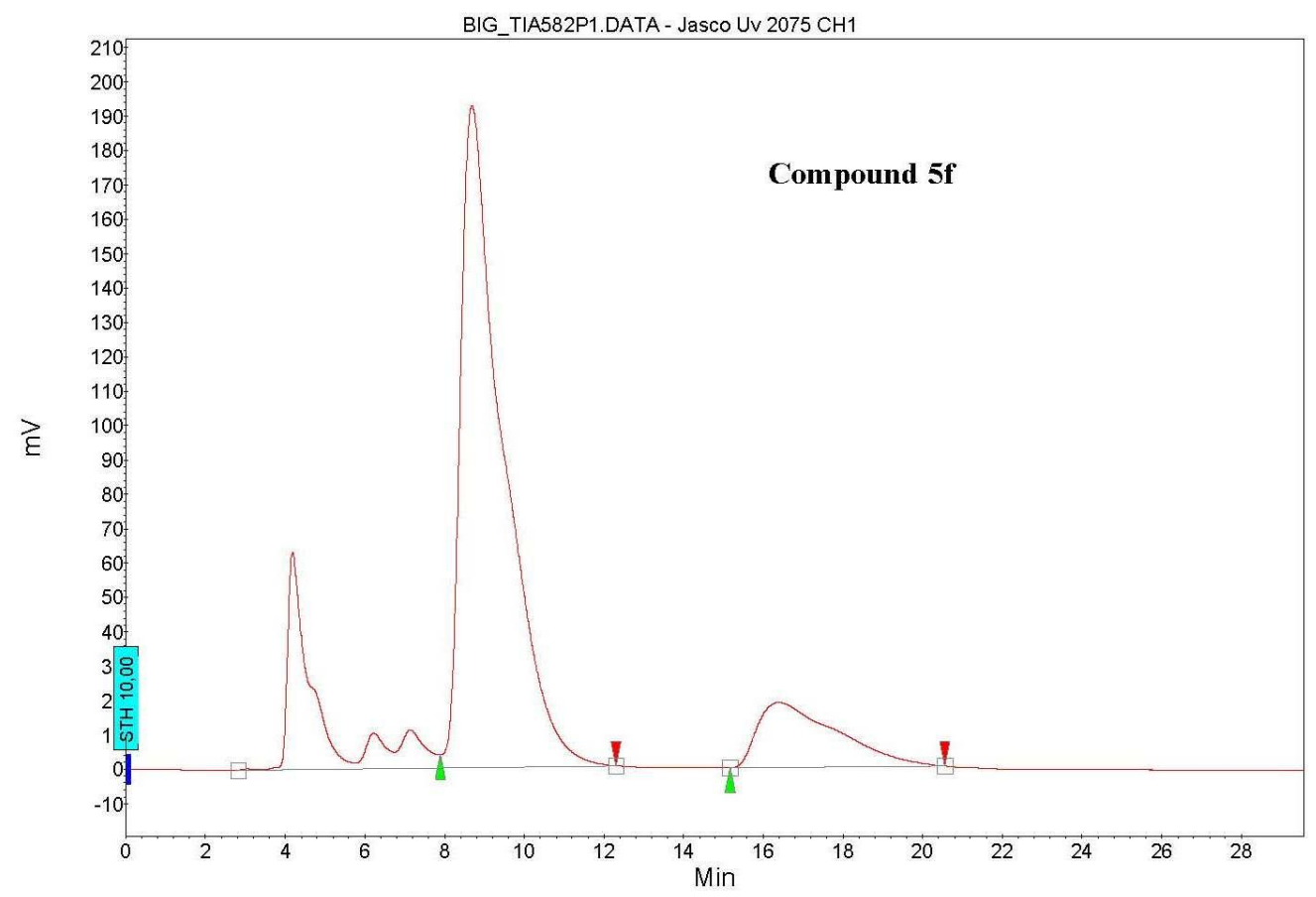

$1 \mathrm{mg}$ in $2 \mathrm{~mL}$ iPrOH

\begin{tabular}{|c|l|r|r|r|r|r|r|r|r|}
\hline Index & Name & Start & Time & End & Ret. time Offset & Quantity & Height & Area & Area \% \\
\hline \hline & & {$[\mathrm{Min}]$} & {$[\mathrm{Min}]$} & {$[\mathrm{Min}]$} & {$[\mathrm{Min}]$} & {$[\%$ Area] } & {$[\mathrm{mV}]$} & {$[\mathrm{mV}$.Min] } & {$[\%]$} \\
\hline 1 & UNKNOWN & 7,892 & 8,692 & 12,300 & 0,000 & 87,13 & 192,5 & 243,7 & 87,129 \\
\hline 2 & UNKNOWN & 15,167 & 16,392 & 20,550 & 0,000 & 12,87 & 18,8 & 36,0 & 12,871 \\
\hline & & & & & & & & & \\
\hline Total & & & & & & 100,00 & 211,3 & 279,7 & 100,000 \\
\hline
\end{tabular}




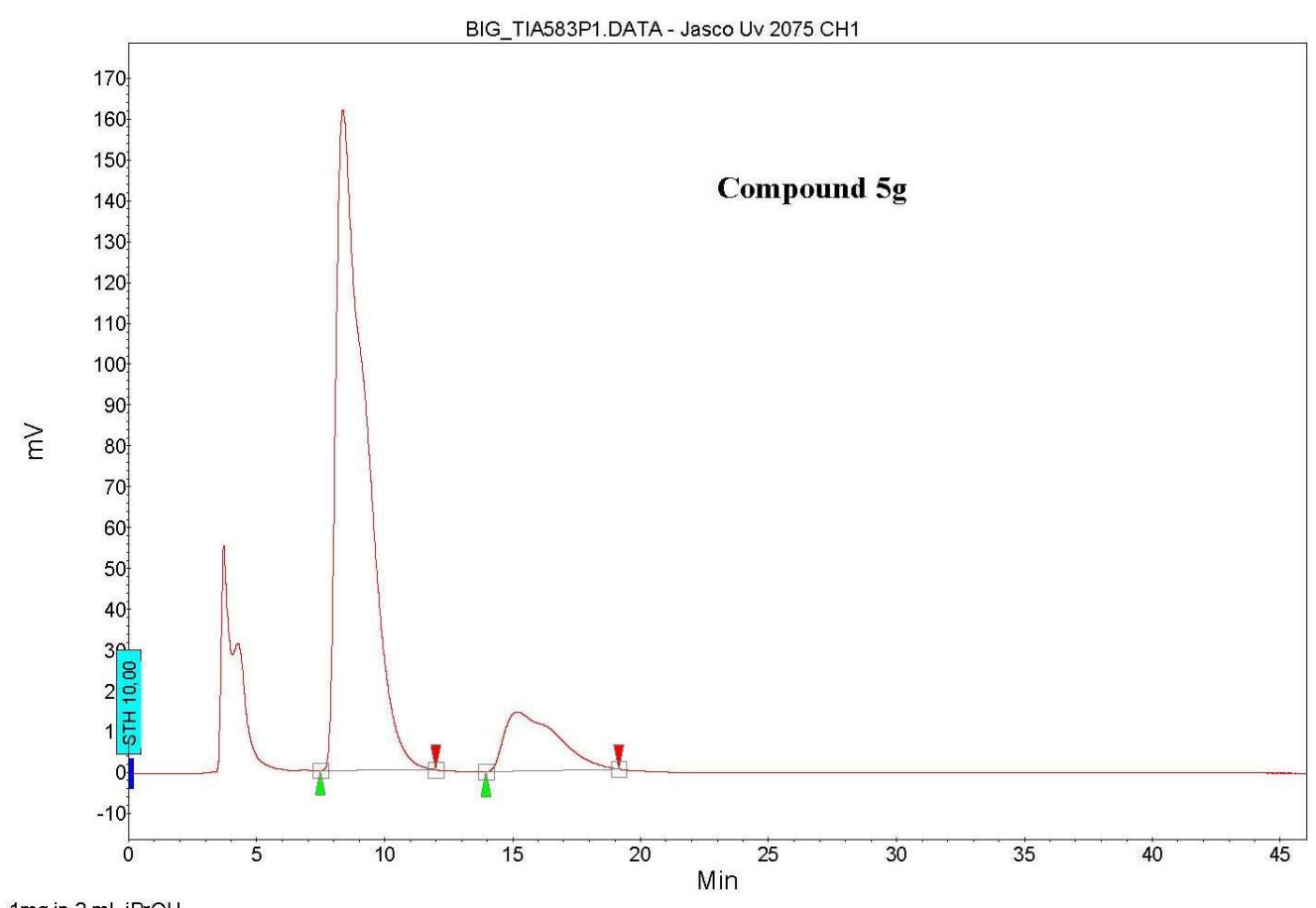

$1 \mathrm{mg}$ in $2 \mathrm{~mL} \mathrm{iPrOH}$

\begin{tabular}{|c|l|r|r|r|r|r|r|r|r|}
\hline Index & Name & Start & Time & End & Ret. time Offset & Quantity & Height & Area & Area \% \\
\hline \hline & & {$[\mathrm{Min}]$} & {$[\mathrm{Min}]$} & {$[\mathrm{Min}]$} & {$[\mathrm{Min}]$} & {$[\%$ Area] } & {$[\mathrm{mV}]$} & {$[\mathrm{mV} . \mathrm{Min}]$} & {$[\%]$} \\
\hline 1 & UNKNOWN & 7,500 & 8,375 & 12,000 & 0,000 & 88,50 & 161,7 & 218,5 & 88,497 \\
\hline 2 & UNKNOWN & 13,967 & 15,192 & 19,150 & 0,000 & 11,50 & 14,5 & 28,4 & 11,503 \\
\hline & & & & & & & & & \\
\hline Total & & & & & & 100,00 & 176,2 & 246,9 & 100,000 \\
\hline
\end{tabular}

BIG_TIA584P1.DATA - Jasco Uv $2075 \mathrm{CH} 1$

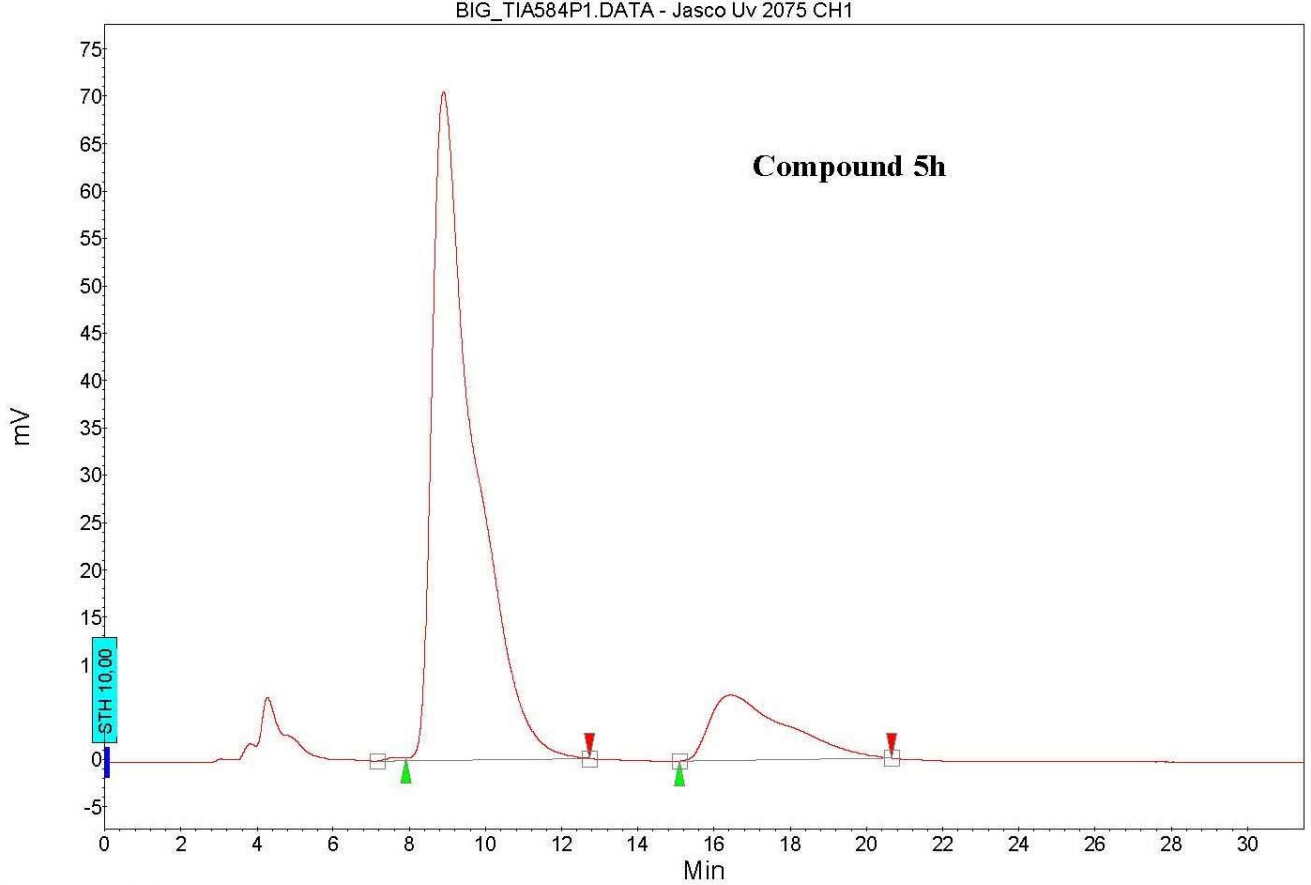

$1 \mathrm{mg}$ in $2 \mathrm{~mL}$ iPrOH

\begin{tabular}{|c|l|r|r|r|r|r|r|r|r|}
\hline Index & Name & Start & Time & End & Ret. time Offset & Quantity & Height & Area & Area \% \\
\hline \hline & & {$[$ Min] } & {$[\mathrm{Min}]$} & {$[\mathrm{Min}]$} & {$[\mathrm{Min}]$} & {$[\%$ Area] } & {$[\mathrm{mV}]$} & {$[\mathrm{mV}$.Min] } & {$[\%]$} \\
\hline 1 & UNKNOWN & 7,917 & 8,900 & 12,733 & 0,000 & 87,55 & 70,6 & 92,1 & 87,548 \\
\hline 2 & UNKNOWN & 15,100 & 16,433 & 20,667 & 0,000 & 12,45 & 6,9 & 13,1 & 12,452 \\
\hline & & & & & & & & & \\
\hline Total & & & & & & 100,00 & 77,5 & 105,2 & 100,000 \\
\hline
\end{tabular}




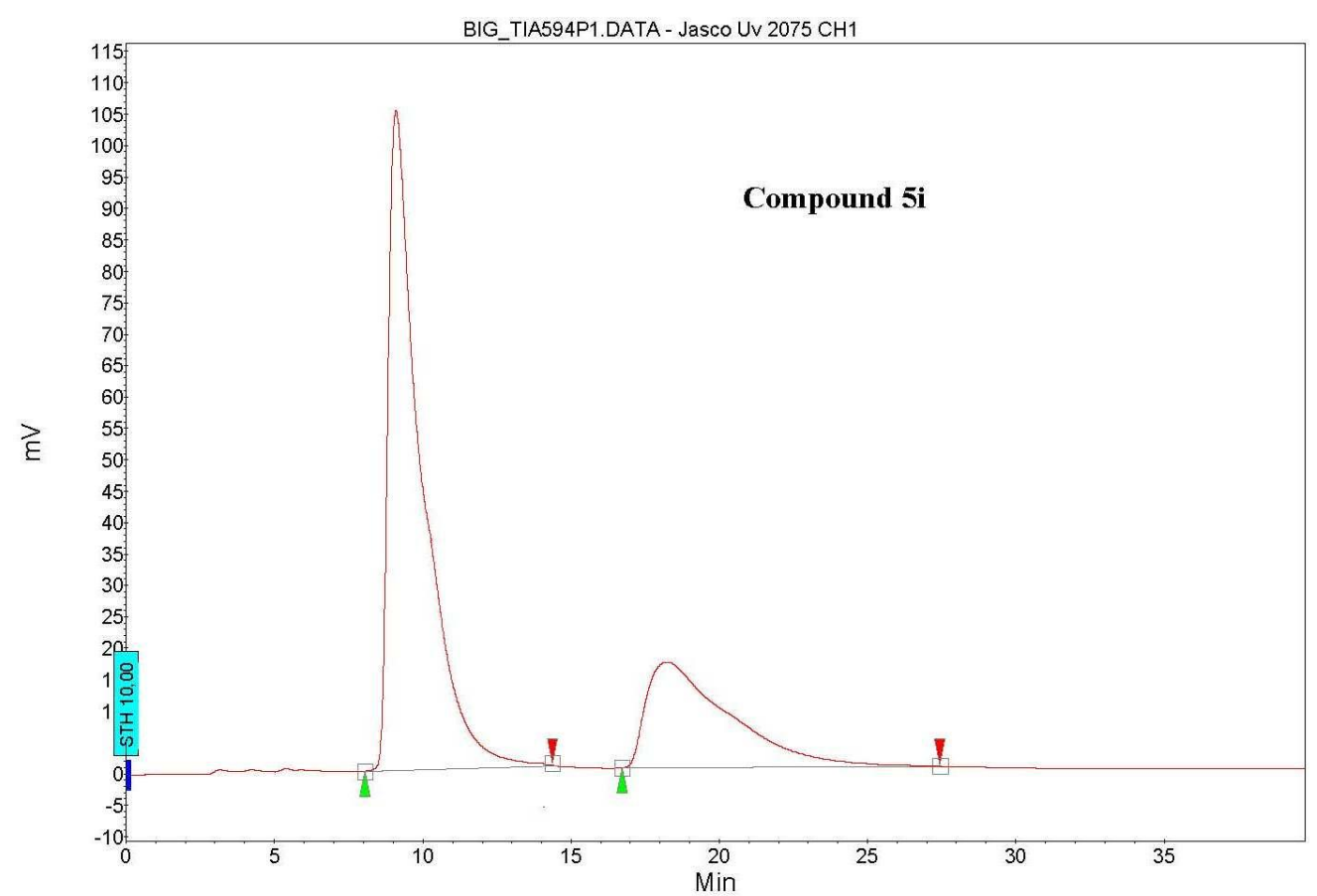

$1 \mathrm{mg}$ in $2 \mathrm{~mL} \mathrm{iPrOH}$

\begin{tabular}{|c|l|r|r|r|r|r|r|r|r|}
\hline Index & Name & Start & Time & End & Ret. time Offset & Quantity & Height & Area & Area \% \\
\hline \hline & & {$[$ Min] } & {$[\mathrm{Min}]$} & {$[\mathrm{Min}]$} & {$[\mathrm{Min}]$} & {$[\%$ Area] } & {$[\mathrm{mV}]$} & {$[\mathrm{mV}$. Min] } & {$[\%]$} \\
\hline 1 & UNKNOWN & 8,050 & 9,150 & 14,550 & 0,000 & 80,48 & 105,1 & 190,5 & 80,482 \\
\hline 2 & UNKNOWN & 16,733 & 18,303 & 27,400 & 0,000 & 19,52 & 16,8 & 46,2 & 19,518 \\
\hline & & & & & & & & & \\
\hline Total & & & & & & 100,00 & 121,9 & 236,7 & 100,000 \\
\hline
\end{tabular}

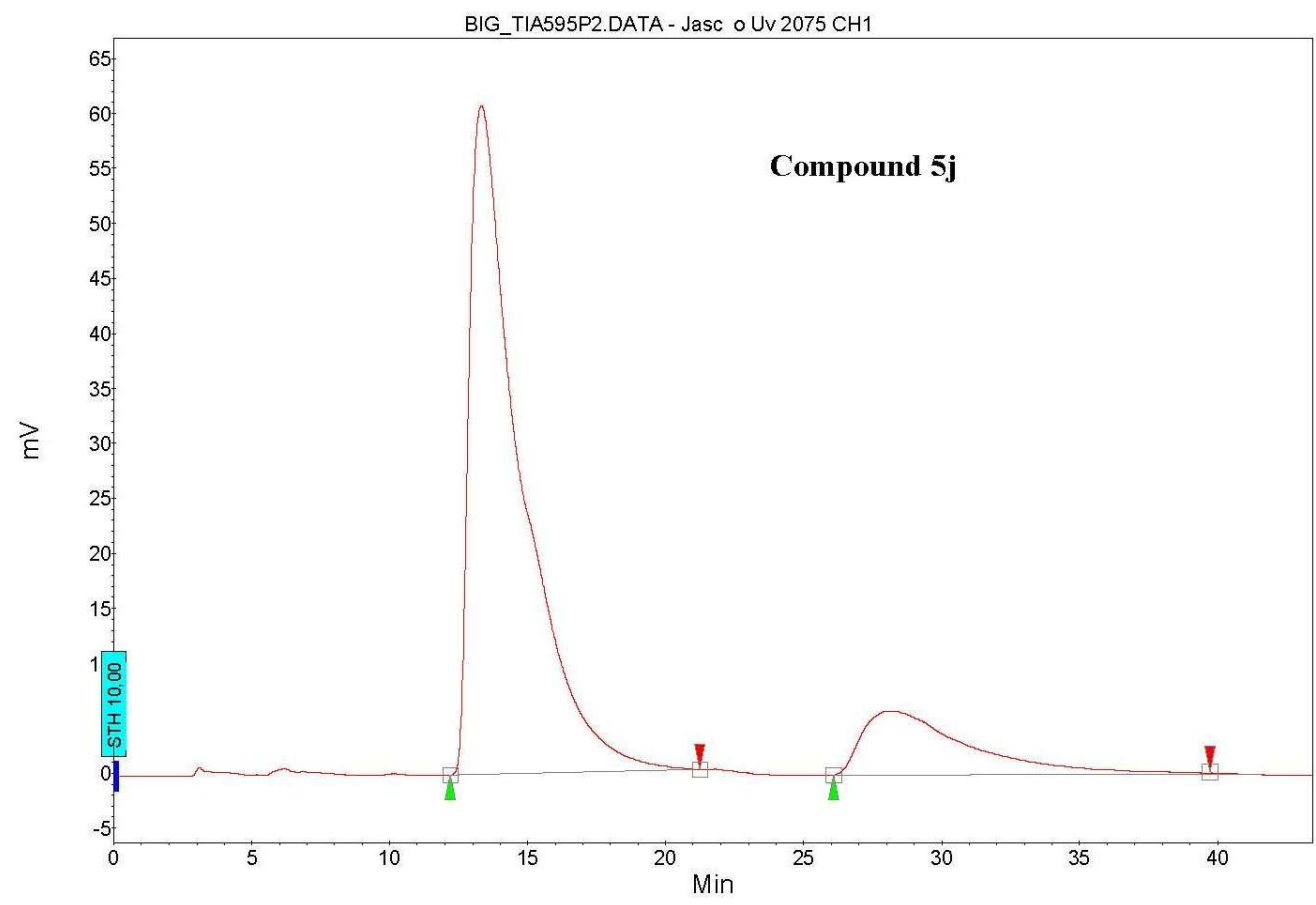

$1 \mathrm{mg}$ in $2 \mathrm{~mL} \mathrm{iPrOH}$

\begin{tabular}{|c|r|r|r|r|r|r|r|r|r|}
\hline Ind ex Name & Start & Time & End & Ret. time Offset & Quantity & Height & Area & Area \% \\
\hline \hline & & {$[$ Min] } & {$[$ Min] } & {$[$ Min] } & {$[$ Min] } & {$[\%$ Area] } & {$[\mathrm{mV}]$} & {$[\mathrm{mV}$. Min] } & {$[\%]$} \\
\hline 1 & UNKNOWN & 12,183 & 13,325 & 21,233 & 0,000 & 86,82 & 60,8 & 157,4 & 86,817 \\
\hline 2 & UNKNOWN & 26,092 & 28,108 & 39,883 & 0,000 & 13,18 & 5,8 & 23,9 & 13,183 \\
\hline & & & & & & & & & \\
\hline Total & & & & & & 100,00 & 66,6 & 181,3 & 100,000 \\
\hline
\end{tabular}


Experimental for X-ray analysis (compound 5h)

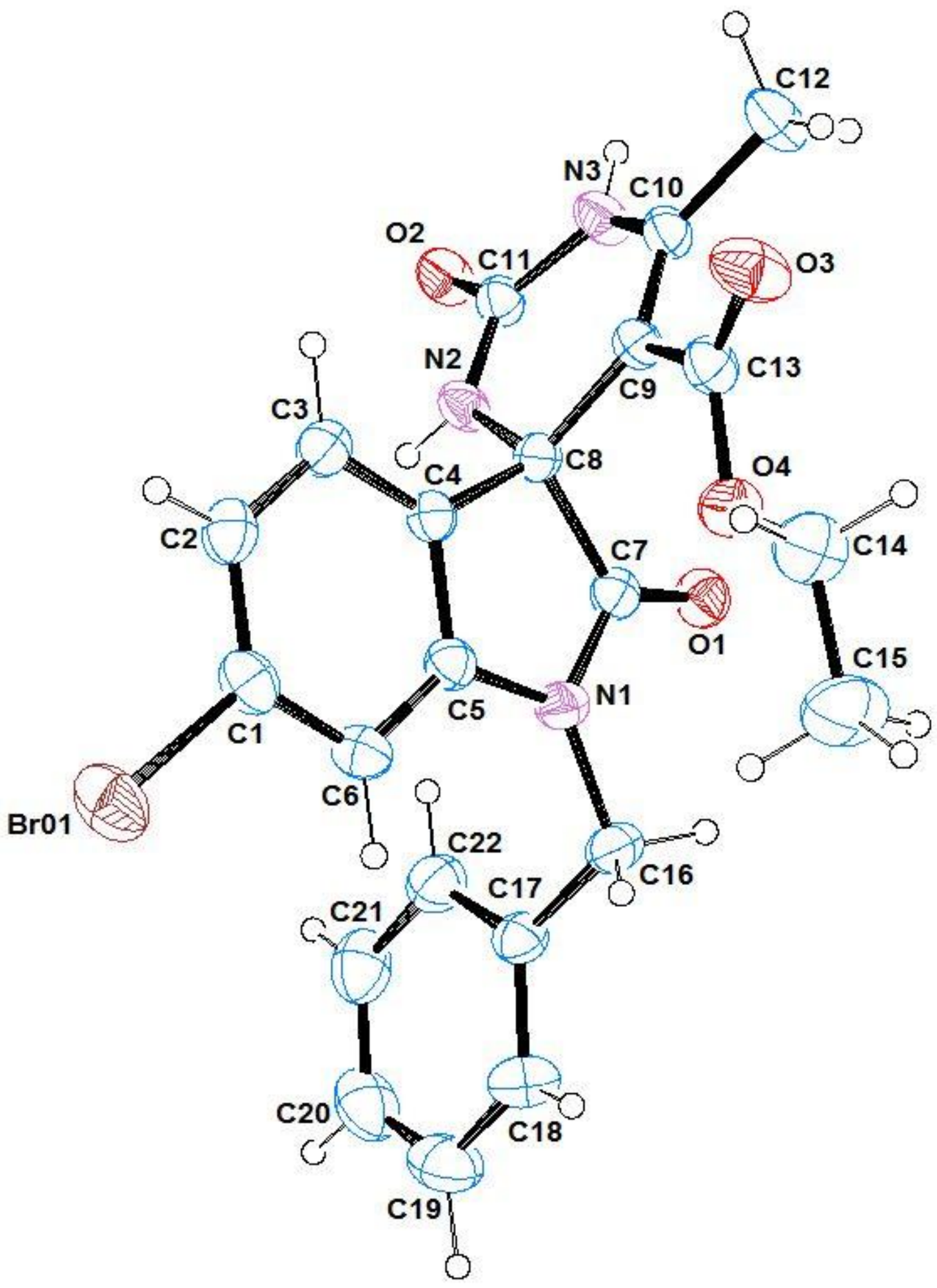

Figure S1: ORTEP ${ }^{1}$ view of $5 \mathrm{~h}$, showing the arbitrary atom-labeling scheme. Atomic displacement parameters for non- $\mathrm{H}$ atoms are at $40 \%$ probability level.

[1] L. J. Farrugia, J. Appl. Crystallogr. 32 (1999) 837. 
CCDC- 1432871 contains the supplementary crystallographic data for this paper. These data can be obtained free of charge via www.ccdc.cam.ac.uk/conts/retrieving.html (or from the Cambridge Crystallographic Data Centre, 12, Union Road, Cambridge CB21EZ, UK; fax: ++44 1223336 033; or deposit@ccdc.cam.ac.uk).

Crystals of $\mathbf{5 h}$ were obtained by slow evaporation of a methanol solution at room temperature, as yellow prisms.

Diffraction data have been collected by means of a Bruker-Axs CCD-based three circle diffractometer, working at ambient temperature with graphite-monochromatized Mo-K $\alpha$ Xradiation $(\lambda=0.71073 \AA)$.

$\mathrm{X}$-ray diffraction data in the $\theta$ range $2-30^{\circ}$ were collected acquiring 4 sets of 600 bidimensional CCD frames with the following operative conditions: omega rotation axis, scan width $0.3^{\circ}$, acquisition time $20 \mathrm{~s}$, sample-to-detector distance $50 \mathrm{~mm}$, phi angle fixed at four different values $\left(40^{\circ}, 100^{\circ}, 160^{\circ}, 330^{\circ}\right)$ for each of the four different sets.

Omega-rotation frames were processed with the SAINT software ${ }^{[\mathrm{X} 1]}$ for data reduction (including intensity integration, background, Lorentz and polarization corrections) and for determination of accurate unit-cell dimensions, obtained by least-squares refinement of the positions of 6222 independent reflections with $\mathrm{I}>10 \sigma(\mathrm{I})$ in the $\theta$ range $2-25^{\circ}$. Absorption effects were empirically evaluated by the SADABS software ${ }^{[\mathrm{X} 2]}$ and absorption correction was applied to the data $(0.658$ and 0.780 min and max transmission factor).

The structure was solved by direct methods ${ }^{[\mathrm{X} 3]}$ and the refinement was carried out with SHELX$97^{[\mathrm{X} 4]}$. All non-H-atoms were refined anisotropically. Hydrogen atoms were detected in a difference Fourier synthesis and refined with isotropic thermal factors. CCDC-1432871 contains the supplementary crystallographic data for this paper. These data can be obtained free of charge via www.ccdc.cam.ac.uk/conts/retrieving.html (or from the Cambridge Crystallographic Data Centre, 12, Union Road, Cambridge CB21EZ, UK; fax: ++44 1223336 033; or deposit@ @cdc.cam.ac.uk).

Crystal data for $5 \mathrm{~h}: \mathrm{C}_{24} \mathrm{H}_{20} \mathrm{~N}_{3} \mathrm{O}_{4} \mathrm{Br}, M_{\mathrm{r}}=472.32 \mathrm{~g} / \mathrm{mol}$, Monoclinic, Space group $\mathrm{P} 2{ }_{1} / \mathrm{a}, a=$ 12.588(3) $\AA, b=11.625(1) \AA, c=15.035(3) \AA, \beta=109.38(1)^{\circ}, V=2075.52(4) \AA^{3}, Z=4, D_{\text {calc }}=$ $1.505 \mathrm{Mg} / \mathrm{m}^{3}, R=0.044(31302$ reflections/6057 obs), $w R 2=0.120, T=293(2) \mathrm{K}, G O F=1.047$. The reflections were collected in the range $2.27^{\circ} \leq \theta \leq 30.02^{\circ}$ employing a $0.24 \times 0.23 \times 0.13$ crystal.

\section{Molecular discussion}

In the solid state, the isatin and the pyrimidine rings are oriented perpendicularly, as shown by the dihedral angle of $89.4(5)^{\circ}$. The pyrimidine ring is characterized by an almost planar conformation, 
as described by the puckering parameters $\mathrm{Q}_{\mathrm{T}}=0.146(2) \AA, \theta^{2}=155(1)^{\circ}$. Atoms $\mathrm{N} 2$ and $\mathrm{C} 8$ have the main deviation out the best mean plane by 0.0767(2) $\AA$ and 0.0954(2) $\AA$, respectively.

The conformation of the lateral chain linked to N1 shows the phenyl ring pointing in the same direction of the pyrimidine carbonyl moiety and its orientation is defined by the torsion angle C5N1-C16-C17 of $75.2(1)^{\circ}$. The benzene ring is perpendicularly oriented with respect to the isatin moiety, forming dihedral angle of $89.0(7)^{\circ}$, while it is slightly inclined with respect to the pyrimidine, with a dihedral angle of $25(1)^{\circ}$.

In the crystal, the intermolecular interactions are characterized by strong centrosymmetric hydrogen bonds between N2-H21 ${ }^{\cdots} \mathrm{O} 2^{\mathrm{I}}\left({ }^{\mathrm{I}}\right.$ at $\left.-\mathrm{x}+1,-\mathrm{y},-\mathrm{z}+1\right)$ at a distance of $2.06(1) \AA$ and angle of $175(1)^{\circ}$, leading to the formation of dimers. These latter are in turns stabilized by $\mathrm{C} \pi-\mathrm{H}$...O contacts involving $\mathrm{O} 2$ and $\mathrm{C} 22-\mathrm{H} 22^{\mathrm{I}}\left({ }^{\mathrm{I}}\right.$ at $\left.-\mathrm{x}+1,-\mathrm{y},-\mathrm{z}+1\right)$. Hydrogen bonds formed by $\mathrm{N} 3-\mathrm{H} 31^{\cdots} \mathrm{O} 1^{\mathrm{II}}\left(^{\mathrm{II}}\right.$ at $\mathrm{x}+1 / 2, \mathrm{y}+1 / 2,-\mathrm{z}+1$ at a distance of $2.13(1) \AA$ and angle of $167(1)^{\circ}$ and weak contacts between O3 and $\mathrm{C} 2-\mathrm{H} 2^{\mathrm{III}}$ (III at $\left.\mathrm{x}+1 / 2,-\mathrm{y}+1 / 2, \mathrm{z}\right)$ and $\mathrm{O} 1$ and $\mathrm{C} 21-\mathrm{H} 211^{\mathrm{IV}}\left({ }^{\mathrm{IV}}\right.$ at $\left.\mathrm{x}+1 / 2,-\mathrm{y}-1 / 2, \mathrm{z}\right)$ consolidated the crystal packing.

[X1] Bruker: SAINT Software Reference Manual. Version 6, Bruker AXS Inc., Madison, Wisconsin, USA, 2003.

[X2] G. M. Sheldrick, TWINABS. University of Göttingen, Göttingen, Germany, 1999.

[X3] A. Altomare, G. Cascarano, C. Giacovazzo, A. Guagliardi, M. C. Burla, G. Polidori, M. Camalli, J. Appl. Cryst. 1994, 27, 435.

[X4] G. M. Sheldrick, Acta Crystallog. Sec. A 2008, A64, 112-122. 


\section{Computational studies}

\section{Determination of the stereochemistry of the $C$-3 quaternary stereocenter}

Mono- and bidimensional NMR analysis on both diastereoisomers $9 \mathbf{a}$ and $\mathbf{9 b}$ allowed to assign each proton and carbon to experimental chemical shifts, although NH protons chemical shifts were not considered. Monte Carlo conformational search was run with Spartan '08 ${ }^{1}$ using MMFF forcefield on both possible stereoisomers with $\left(3 S, 1^{\prime} S\right)$ and $\left(3 R, 1^{\prime} S\right)$ absolute configurations, with standard parameters and convergence criteria. All structures with population $>1 \%$ were further optimized with GAUSSIAN09 ${ }^{2}$ at quantum-mechanics DFT/B3LYP/6-31g(d,p) in gas phase, followed by single-point $a b$ initio calculations of energy (DFT/B3LYP/6-31g(d,p)) and GIAO shielding constants at the DFT/6-311+G(2d,p)/SCRF-dmso level. Boltzmann averaging over the conformers, followed by linear regression employing reported ${ }^{3}{ }^{1} \mathrm{H}$ and ${ }^{13} \mathrm{C}$ NMR scaling factors afforded theoretical NMR chemical shift for the two possible $\left(3 S, 1^{\prime} S\right)$ and $\left(3 R, 1^{\prime} S\right)$ absolute configurations. Comparison parameters 3 (CP3) were computed for protons, carbons and all data. Application of the Bayes' theorem afforded the percentage probability for each assignment. ${ }^{4}$

\begin{tabular}{lcccc} 
Proton & $\begin{array}{c}\text { Theoretical } \\
\left(3 R, 1^{\prime} S\right)\end{array}$ & $\begin{array}{c}\text { Theoretical } \\
\left(3 S, 1^{\prime} S\right)\end{array}$ & $\begin{array}{c}\text { Exp. } \\
\mathbf{9 a}\end{array}$ & $\begin{array}{c}\text { Exp. } \\
\mathbf{9 b}\end{array}$ \\
\hline $\mathrm{H} 4$ & 7,250 & 7,383 & 7,33 & 7,31 \\
$\mathrm{H} 5$ & 7,009 & 6,988 & 7,00 & 7,00 \\
$\mathrm{H} 6$ & 7,139 & 7,073 & 7,17 & 7,17 \\
$\mathrm{H} 7$ & 6,397 & 6,367 & 6,63 & 6,57 \\
$\mathrm{H}_{\mathrm{o}}(\mathrm{Bn})$ & 7,778 & 7,755 & 7,46 & 7,42 \\
$\mathrm{H}_{\mathrm{m}}(\mathrm{Bn})$ & 7,322 & 7,321 & 7,28 & 7,25 \\
$\mathrm{H}_{\mathrm{p}}(\mathrm{Bn})$ & 7,279 & 7,266 & 7,26 & 7,24 \\
$\mathrm{H} 10$ & 4,762 & 4,719 & 4,78 & 4,77 \\
$\mathrm{H} 14$ & 2,098 & 1,907 & 1,91 & 2,01 \\
$\mathrm{H}^{\prime}$ & 5,189 & 4,783 & 4,66 & 4,75 \\
$\mathrm{H}^{\prime}$ & 1,316 & 1,067 & 1,04 & 1,32 \\
$\mathrm{H}^{\prime}{ }_{\mathrm{o}}(\mathrm{Ph})$ & 7,034 & 7,369 & 7,25 & 6,83 \\
$\mathrm{H}^{\prime}{ }_{\mathrm{m}}(\mathrm{Ph})$ & 7,236 & 7,370 & 7,27 & 7,11 \\
$\mathrm{H}^{\prime}{ }_{\mathrm{p}}(\mathrm{Ph})$ & 7,188 & 7,306 & 7,19 & 7,09
\end{tabular}

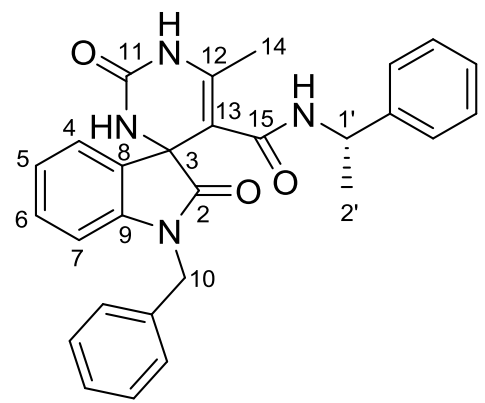

\footnotetext{
${ }^{1}$ Spartan'08. Wavefunction, Inc. Irvine, CA.

${ }^{2}$ Frisch, M. J.; Trucks, G. W.; Schlegel, H. B.; Scuseria, G. E.; Robb, M. A.; Cheeseman, J. R.; Scalmani, G.; Barone, V.; Mennucci, B.; Petersson, G. A.; Nakatsuji, H.; Caricato, M.; Li, X.; Hratchian, H. P.; Izmaylov, A. F.; Bloino, J.; Zheng, G.; Sonnenberg, J. L.; Hada, M.; Ehara, M.; Toyota, K.; Fukuda, R.; Hasegawa, J.; Ishida, M.; Nakajima, T.; Honda, Y.; Kitao, O.; Nakai, H.; Vreven, T.; Montgomery, J. A., Jr.; Peralta, J. E.; Ogliaro, F.; Bearpark, M.; Heyd, J. J.; Brothers, E.; Kudin, K. N.; Staroverov, V. N.; Kobayashi, R.; Normand, J.; Raghavachari, K.; Rendell, A.; Burant, J. C.; Iyengar, S. S.; Tomasi, J.; Cossi, M.; Rega, N.; Millam, J. M.; Klene, M.; Knox, J. E.; Cross, J. B.; Bakken, V.; Adamo, C.; Jaramillo, J.; Gomperts, R.; Stratmann, R. E.; Yazyev, O.; Austin, A. J.; Cammi, R.; Pomelli, C.; Ochterski, J. W.; Martin, R. L.; Morokuma, K.; Zakrzewski, V. G.; Voth, G. A.; Salvador, P.; Dannenberg, J. J.; Dapprich, S.; Daniels, A. D.; Farkas, Ö.; Foresman, J. B.; Ortiz, J. V.; Cioslowski, J.; Fox, D. J. Gaussian 09, Revision A.02, Gaussian, Inc., Wallingford, CT, 2009.

${ }^{3}$ Pierens, G. K. J. Comp. Chem. 2014, 35, 1388.

${ }^{4}$ Smith, S. G.; Goodman, J. M. J. Org. Chem. 2009, 74, 4597.
} 


\begin{tabular}{|c|c|c|c|c|}
\hline Carbon & $\begin{array}{c}\text { Theoretical } \\
\left(3 R, 1^{\prime} S\right)\end{array}$ & $\begin{array}{c}\text { I Theoretical } \\
\left(3 S, 1^{\prime} S\right)\end{array}$ & $\begin{array}{l}\text { Exp. } \\
9 a\end{array}$ & $\begin{array}{l}\text { Exp. } \\
9 b\end{array}$ \\
\hline $\mathrm{C} 2$ & 175,9154 & 175,8929 & 177,4 & 177,44 \\
\hline C3 & 66,3331 & 66,5677 & 64,341 & 64,46 \\
\hline $\mathrm{C} 8$ & 131,0964 & 131,2399 & 132,37 & 132,69 \\
\hline $\mathrm{C} 4$ & 123,1457 & 122,5555 & 125,29 & 125,33 \\
\hline $\mathrm{C} 5$ & 120,7829 & 120,4989 & 123,04 & 123,18 \\
\hline $\mathrm{C} 6$ & 128,5257 & 127,8360 & 130,17 & 130,12 \\
\hline $\mathrm{C} 7$ & 108,2972 & 108,5746 & 109,94 & 109,97 \\
\hline C9 & 143,3782 & 144,1032 & 144,42 & 144,32 \\
\hline C10 & 43,7500 & 43,6839 & 44,23 & 44,24 \\
\hline$C_{0}(B n)$ & 126,2063 & 125,9411 & 128,33 & 128,24 \\
\hline$C_{m}(B n)$ & 126,6883 & 126,8280 & 129,41 & 129,36 \\
\hline$C_{p}(B n)$ & 125,4642 & 125,4950 & 128,15 & 128,07 \\
\hline$C(B n)$ & 137,0134 & 137,1675 & 137,3 & 137,27 \\
\hline C11 & 148,7539 & 148,4746 & 153,25 & 153,03 \\
\hline C12 & 142,1279 & 142,4865 & 138 & 138,35 \\
\hline C13 & 105,2285 & 105,4434 & 105,12 & 105,123 \\
\hline C14 & 17,0658 & 16,8558 & 18,31 & 18,36 \\
\hline C15 & 163,4681 & 163,1576 & 165,616 & 165,57 \\
\hline$C 1^{\prime}$ & 47,5551 & 51,3586 & 48,61 & 48,127 \\
\hline$C 2^{\prime}$ & 16,7350 & 17,8353 & 23,04 & 22,52 \\
\hline $\mathrm{C}_{0}^{\prime}(\mathrm{Ph})$ & 125,6453 & 125,7907 & 127,231 & 126,78 \\
\hline $\mathrm{C}_{\mathrm{m}}^{\prime}(\mathrm{Ph})$ & 126,3054 & 126,9164 & 129,26 & 128,9 \\
\hline $\mathrm{C}_{\mathrm{p}}^{\prime}(\mathrm{Ph})$ & 125,2688 & 126,0116 & 127,65 & 127,14 \\
\hline $\mathrm{C}^{\prime}(\mathrm{Ph})$ & 142,7919 & 142,4945 & 145,31 & 144,88 \\
\hline Assignment & $\begin{array}{c}\mathrm{CP3} \\
\text { (Proton) }\end{array}$ & $\begin{array}{c}\text { CP3 } \\
\text { (Carbon) }\end{array}$ & $\begin{array}{l}\text { CP3 } \\
\text { (All) }\end{array}$ & \\
\hline $\begin{array}{l}\left(3 S, 1^{\prime} S\right)-9 \mathbf{a} \\
\left(3 R, 1^{\prime} S\right)-9 \mathbf{b}\end{array}$ & 0,773 & 0,104 & 0,137 & \\
\hline $\begin{array}{l}\left(3 S, 1^{\prime} S\right)-9 \mathbf{b} \\
\left(3 R, 1^{\prime} S\right)-9 \mathbf{a}\end{array}$ & $-0,951$ & $-1,917$ & $-1,64$ & \\
\hline Assignment & $\begin{array}{c}\text { Probability } \\
\text { on proton } \\
\text { data }(\%)\end{array}$ & $\begin{array}{l}\text { Probability } \\
\text { on carbon } \\
\text { data (\%) }\end{array}$ & $\begin{array}{c}\text { Probability } \\
\text { on all data } \\
(\%)\end{array}$ & \\
\hline $\begin{array}{l}\left(3 S, 1^{\prime} S\right)-9 \mathrm{a} \\
\left(3 R, 1^{\prime} S\right)-9 \mathrm{~b}\end{array}$ & 100 & 100 & 100 & \\
\hline $\begin{array}{l}\left(3 S, 1^{\prime} S\right)-9 \mathrm{~b} \\
\left(3 R, 1^{\prime} S\right)-9 \mathrm{a}\end{array}$ & 0 & 0 & 0 & \\
\hline
\end{tabular}




\section{$\underline{\text { Transition state }}$}

All the reaction components were first submitted to a Monte Carlo conformational search with MM (MMFF94 force field) minimization. The global minima were then submitted to a geometry optimization with DFT at the B3LYP/6-31G(d) level. All the minima were confirmed by frequency calculation. Transition state search was first performed with HF/3-21G on a simplified model (figure) and then refined with DFT B3LYP/6-31G(d). Single point calculation at the B3LYP/6$31 \mathrm{G}(\mathrm{d}, \mathrm{p})$ level was then performed on the complete TS structures. TS were confirmed by analysis of the imaginary frequencies.

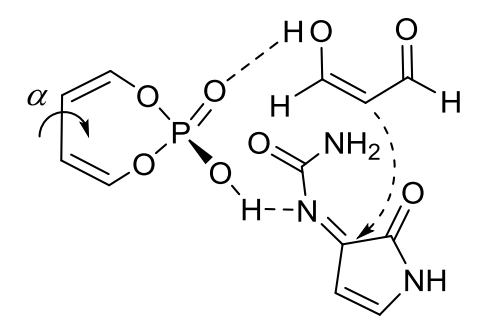

Figure. Simplified model for the transition state search. A constrained dihedral angle $\alpha=-59.8^{\circ}$ was used.

\section{TS-A}

$\mathrm{E}=-3266.97977$ Hartrees; $\mathrm{ZPE}=2282.70 \mathrm{~kJ} / \mathrm{mol}$

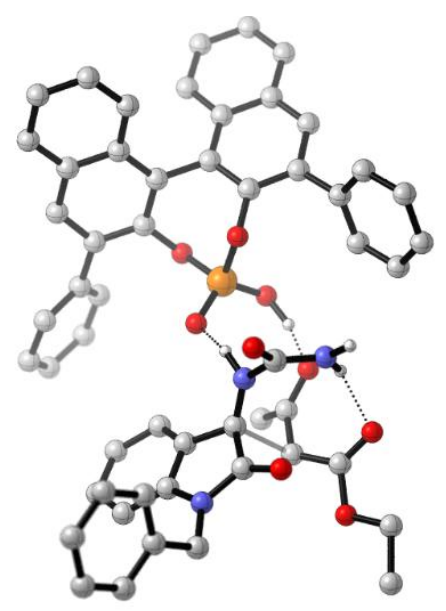

Cartesian Coordinates (Angstroms)

$$
\begin{array}{lllll}
1 & \mathrm{C} & -1.8892290 & 1.7792323 & -1.5767275 \\
2 & \mathrm{O} & -1.9099040 & 1.3892922 & -2.7191745 \\
3 & \mathrm{~N} & -1.8670942 & 0.8156962 & -0.4775048 \\
4 & \mathrm{~N} & -1.7997383 & 3.0374635 & -1.1551571 \\
5 & \mathrm{H} & -1.8329944 & 3.7591594 & -1.8424761
\end{array}
$$




$\begin{array}{lllll}6 & \mathrm{C} & -2.8887838 & 0.3864052 & 0.2242179 \\ 7 & \mathrm{H} & -1.8601058 & 3.2953184 & -0.1879628 \\ 8 & \mathrm{C} & -3.7709850 & 2.9042266 & 1.6067527 \\ 9 & \mathrm{O} & -3.1609968 & 3.8602303 & 1.1563790 \\ 10 & \mathrm{C} & -3.2091039 & 1.6255850 & 2.0134133 \\ 11 & \mathrm{H} & -3.8742699 & 0.9906672 & 2.5626707 \\ 12 & \mathrm{C} & -1.8407773 & 1.5213665 & 2.3481786 \\ 13 & \mathrm{O} & -0.9227088 & 2.2404134 & 1.9121698 \\ 14 & \mathrm{H} & 0.4863183 & 1.7950655 & 2.0951394 \\ 15 & \mathrm{P} & 1.4975814 & 0.2367059 & 0.9733152 \\ 16 & \mathrm{O} & 1.3669767 & 1.2612068 & 2.0681876 \\ 17 & \mathrm{O} & 0.2479051 & -0.4901086 & 0.6081588 \\ 18 & \mathrm{H} & -0.9758390 & 0.3497510 & -0.2809162 \\ 19 & \mathrm{O} & 2.1653719 & 0.9088527 & -0.3736185 \\ 20 & \mathrm{O} & 2.6414851 & -0.8479806 & 1.4436987 \\ 21 & \mathrm{C} & 3.4793745 & 1.3088645 & -0.3576033 \\ 22 & \mathrm{C} & 4.4738249 & 0.3086206 & -0.2823161 \\ 23 & \mathrm{C} & 3.1983978 & -1.6886087 & 0.5133777 \\ 24 & \mathrm{C} & 4.0725981 & -1.1197153 & -0.4370685 \\ 25 & \mathrm{C} & -4.2780130 & 0.5947345 & -0.3961390 \\ 26 & \mathrm{C} & -3.0154971 & -1.0312558 & 0.7574272 \\ 27 & \mathrm{C} & -4.2921838 & -1.5182674 & 0.4423307 \\ 28 & \mathrm{~N} & -5.0334024 & -0.5492690 & -0.2446699 \\ 29 & \mathrm{O} & -4.6133988 & 1.6393854 & -0.9376629 \\ 30 & \mathrm{C} & -2.1673153 & -1.7862926 & 1.5266990 \\ 31 & \mathrm{H} & -1.2207507 & -1.3750714 & 1.8490764 \\ 32 & \mathrm{C} & -4.7100993 & -2.7861951 & 0.8235505 \\ 33 & \mathrm{H} & -5.6879970 & -3.1665981 & 0.5615406 \\ 34 & \mathrm{C} & -3.8307415 & -3.5657177 & 1.5757999 \\ 35 & \mathrm{H} & -4.1205165 & -4.5590134 & 1.9018997 \\ 36 & \mathrm{C} & -2.5742873 & -3.0601655 & 1.9366622 \\ 39 & \mathrm{H} & -1.8956681 & -3.6388924 & 2.5545045 \\ \mathrm{C} & 5.8342864 & 0.6978946 & -0.0802179 \\ & \mathrm{C} & 8.5129522 & 1.5604550 & 0.2967438\end{array}$




\begin{tabular}{|c|c|c|c|c|}
\hline 40 & $\mathrm{C}$ & 6.8911152 & -0.2143039 & 0.1667434 \\
\hline & $\mathrm{C}$ & 6.1640407 & 2.0721529 & -0.1116004 \\
\hline & $\mathrm{C}$ & 7.4954572 & 2.4852979 & 0.0701582 \\
\hline & $\mathrm{C}$ & 8.2099514 & 0.2079731 & 0.3489551 \\
\hline & $\mathrm{H}$ & 6.6817561 & -1.2798169 & 0.2398993 \\
\hline & $\mathrm{H}$ & 7.7431242 & 3.5439276 & 0.0479613 \\
\hline & $\mathrm{H}$ & 8.9877252 & -0.5243496 & 0.5420936 \\
\hline & $\mathrm{H}$ & 9.5330454 & 1.8993094 & 0.4457855 \\
\hline & $\mathrm{C}$ & 4.5437699 & -1.9320065 & -1.5135754 \\
\hline 4 & $\mathrm{C}$ & 5.4680070 & -3.6287121 & -3.5944840 \\
\hline 5 & $\mathrm{C}$ & 5.3031687 & -1.4400799 & -2.6050714 \\
\hline 5 & $\mathrm{C}$ & 4.2340124 & -3.3109904 & -1.5143331 \\
\hline 5 & $\mathrm{C}$ & 4.7062501 & -4.1417559 & -2.5464417 \\
\hline 5 & $\mathrm{C}$ & 5.7631133 & -2.2740067 & -3.6269537 \\
\hline 5 & $\mathrm{H}$ & 5.5255190 & -0.3769109 & -2.6752246 \\
\hline 55 & $\mathrm{H}$ & 4.4645000 & -5.2019210 & -2.5444193 \\
\hline 56 & $\mathrm{H}$ & 6.3381735 & -1.8544694 & -4.4469483 \\
\hline 57 & $\mathrm{H}$ & 5.8118327 & -4.2829236 & -4.3890750 \\
\hline 58 & $\mathrm{C}$ & 5.1588047 & 3.0237323 & -0.3321751 \\
\hline 59 & $\mathrm{H}$ & 5.4179786 & 4.0802784 & -0.3710943 \\
\hline 6 & $\mathrm{C}$ & 3.8051627 & 2.6697086 & -0.4665060 \\
\hline 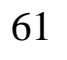 & $\mathrm{C}$ & 3.4540664 & -3.8507178 & -0.4800880 \\
\hline 62 & $\mathrm{H}$ & 3.2499474 & -4.9194626 & -0.4777129 \\
\hline 63 & $\mathrm{C}$ & 2.9099878 & -3.0617260 & 0.5497967 \\
\hline 64 & $\mathrm{O}$ & -5.1412978 & 2.9330513 & 1.7698927 \\
\hline 65 & $\mathrm{C}$ & -5.7432007 & 4.1226198 & 1.2662198 \\
\hline 6 & $\mathrm{H}$ & -5.4055789 & 4.9856495 & 1.8509770 \\
\hline 67 & $\mathrm{H}$ & -5.4824488 & 4.2647568 & 0.2110771 \\
\hline 68 & $\mathrm{C}$ & -7.2477355 & 3.9792765 & 1.3956200 \\
\hline 69 & $\mathrm{H}$ & -7.5316728 & 3.8199695 & 2.4411385 \\
\hline 70 & $\mathrm{H}$ & -7.6010397 & 3.1084330 & 0.8332650 \\
\hline 71 & $\mathrm{H}$ & -7.7582750 & 4.8715805 & 1.0227852 \\
\hline 72 & $\mathrm{C}$ & -6.3766433 & -0.6868792 & -0.7254693 \\
\hline 7 & $\mathrm{H}$ & -6.7656873 & 0.2972868 & -1.0136476 \\
\hline
\end{tabular}




\begin{tabular}{|c|c|c|c|c|}
\hline 74 & $\mathrm{H}$ & -6.9933618 & -1.0380766 & 0.1109966 \\
\hline & $\mathrm{C}$ & -6.5269602 & -1.6097703 & -1.9173111 \\
\hline & $\mathrm{C}$ & -6.8707722 & -3.2846897 & $-4.147570 \mathrm{C}$ \\
\hline & $\mathrm{C}$ & -7.6550348 & -2.4386889 & -2.0237025 \\
\hline & $\mathrm{C}$ & -5.5859495 & -1.6181359 & -2.958154 \\
\hline & $\mathrm{C}$ & -5.7534122 & -2.4569667 & -4.0612412 \\
\hline & $\mathrm{C}$ & -7.8235570 & -3.2737747 & -3.1306456 \\
\hline & $\mathrm{H}$ & -8.4077628 & -2.4395499 & -1.2392114 \\
\hline 8 & $\mathrm{H}$ & -4.7037959 & -0.9770918 & -2.9132388 \\
\hline 83 & $\mathrm{H}$ & -5.0013630 & -2.4588944 & -4.8463577 \\
\hline 84 & $\mathrm{H}$ & -8.6970461 & -3.9157459 & -3.1982489 \\
\hline 85 & $\mathrm{H}$ & -6.9952060 & -3.9362139 & -5.0073081 \\
\hline 86 & $\mathrm{C}$ & 2.8220668 & 3.7321524 & $-0.748435 c$ \\
\hline 87 & $\mathrm{C}$ & 0.9717824 & 5.7944104 & -1.3024426 \\
\hline 88 & $\mathrm{C}$ & 1.7749504 & 4.0195656 & 0.1306942 \\
\hline 89 & $\mathrm{C}$ & 2.9250739 & 4.5118245 & -1.9166716 \\
\hline 90 & $\mathrm{C}$ & 2.0108093 & 5.5346751 & -2.1918748 \\
\hline 91 & $\mathrm{C}$ & 0.8536133 & 5.0338770 & -0.1432627 \\
\hline 92 & $\mathrm{H}$ & 1.6361609 & 3.4270533 & 1.0355368 \\
\hline 93 & $\mathrm{H}$ & 3.7133796 & 4.2998394 & -2.6335296 \\
\hline 94 & $\mathrm{H}$ & 2.0968038 & 6.1062277 & -3.1094513 \\
\hline 95 & $\mathrm{H}$ & 0.0254164 & 5.1770840 & 0.5488882 \\
\hline 96 & $\mathrm{H}$ & 0.2360398 & 6.5617198 & -1.5162299 \\
\hline 97 & $\mathrm{C}$ & 2.1153528 & -3.7103866 & 1.6189545 \\
\hline 98 & $\mathrm{C}$ & 0.6846913 & -5.0485907 & 3.6602892 \\
\hline 99 & $\mathrm{C}$ & 1.1306233 & -4.6683358 & 1.3159208 \\
\hline 1100 & $\mathrm{C}$ & 2.3450200 & -3.4301366 & 2.9761523 \\
\hline 101 & $\mathrm{C}$ & 1.6350548 & -4.0860581 & 3.9866031 \\
\hline 102 & $\mathrm{C}$ & 0.4307720 & -5.3397840 & 2.3233169 \\
\hline 103 & $\mathrm{H}$ & 0.8758818 & -4.8681635 & 0.2774993 \\
\hline 104 & $\mathrm{H}$ & 3.0676500 & -2.6656681 & 3.2563595 \\
\hline 105 & $\mathrm{H}$ & 1.8105637 & -3.8136696 & 5.0228883 \\
\hline 106 & $\mathrm{H}$ & -0.3401559 & -6.0546744 & 2.0549799 \\
\hline 107 & $\mathrm{H}$ & 0.1164804 & -5.5370571 & 4.444662 \\
\hline
\end{tabular}




$\begin{array}{lllll}108 & \mathrm{C} & -1.5317760 & 0.6815223 & 3.6474475 \\ 109 & \mathrm{H} & -1.4319744 & 1.3617462 & 4.4996729 \\ 110 & \mathrm{H} & -0.6086860 & 0.1059486 & 3.5385628 \\ 111 & \mathrm{H} & -2.3522032 & -0.0098574 & 3.8568810\end{array}$

\section{TS-B}

$\mathrm{E}=-3266.89864$ Hartrees; ZPE $=2278.24 \mathrm{~kJ} / \mathrm{mol}$

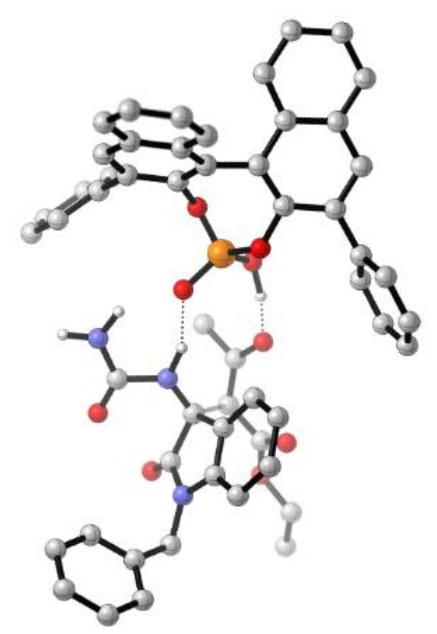

Cartesian Coordinates (Angstroms)

$\begin{array}{lllll}1 & \mathrm{C} & -2.426682 & -2.408128 & 0.707203 \\ 2 & \mathrm{O} & -3.554436 & -2.830458 & 0.852490 \\ 3 & \mathrm{~N} & -2.185059 & -1.004436 & 0.593342 \\ 4 & \mathrm{~N} & -1.288257 & -3.099359 & 0.628425 \\ 5 & \mathrm{H} & -1.327048 & -4.093185 & 0.674452 \\ 6 & \mathrm{C} & -3.153860 & -0.135645 & 0.711594 \\ 7 & \mathrm{H} & -0.408886 & -2.619520 & 0.535719 \\ 8 & \mathrm{C} & -4.165168 & 1.946489 & 2.195336 \\ 9 & \mathrm{O} & -3.821716 & 2.625795 & 1.235846 \\ 10 & \mathrm{C} & -3.401378 & 0.875460 & 2.794472 \\ 11 & \mathrm{H} & -3.918982 & 0.255830 & 3.500374 \\ 12 & \mathrm{C} & -2.012392 & 0.884222 & 2.876246 \\ 13 & \mathrm{O} & -1.212344 & 1.591040 & 2.204959 \\ 14 & \mathrm{H} & 0.126850 & 1.342882 & 2.228921 \\ 15 & \mathrm{P} & 1.546666 & 0.031600 & 0.982235\end{array}$




\begin{tabular}{|c|c|c|c|c|}
\hline 6 & $\mathrm{O}$ & 477 & -0 & 15 \\
\hline 17 & $\mathrm{H}$ & -1.206230 & -0.722114 & 0.483098 \\
\hline & $\mathrm{O}$ & 1.126355 & 0.956156 & 2.132304 \\
\hline & $\mathrm{O}$ & 2.706239 & -0.816709 & 1.303235 \\
\hline 20 & $\mathrm{O}$ & 2.189006 & 1.047592 & -0.308464 \\
\hline & $r$ & 3.595844 & -1.466715 & 0.439669 \\
\hline 2 & $\mathrm{C}$ & 4.354521 & -0.760966 & -0 \\
\hline 2 & $\mathrm{C}$ & 4.528990 & 0.671834 & 761 \\
\hline 2 & $\mathrm{C}$ & 3.504398 & 1.526216 & 8939 \\
\hline 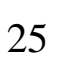 & $\mathrm{C}$ & 5.837748 & 1.249351 & 3883 \\
\hline 26 & $\mathrm{C}$ & 8.401498 & 2.449992 & 6915 \\
\hline 27 & $\mathrm{C}$ & 7.001656 & 0.490350 & 0.179537 \\
\hline 28 & $\mathrm{C}$ & 6.01 & 2.656 & 0085 \\
\hline 2 & $\mathrm{C}$ & 7.283583 & 3.235531 & -0.027025 \\
\hline J & $\mathrm{C}$ & 8.259666 & 1.076059 & 0.347346 \\
\hline 5 & $\mathrm{H}$ & 6.942713 & -0.590977 & 0.291515 \\
\hline 5 & $\mathrm{H}$ & 7.409665 & 4.314456 & -0.093823 \\
\hline 33 & $\mathrm{H}$ & 9.122210 & 0.452142 & 0.566260 \\
\hline 34 & $\mathrm{H}$ & 9.375933 & 2.912276 & 790 \\
\hline 35 & $\mathrm{C}$ & 3.557890 & -2.883824 & 0.486613 \\
\hline 36 & $\mathrm{C}$ & 4.295254 & -3.586254 & -0.469132 \\
\hline$J$ & $\mathrm{C}$ & 3.631238 & 2.929473 & -0.504365 \\
\hline 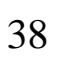 & $\mathrm{C}$ & 4.909892 & 3.476290 & -0.421681 \\
\hline 39 & $\mathrm{H}$ & 5.0 & 35 & -0.486406 \\
\hline 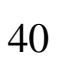 & $\mathrm{C}$ & 5.0 & -1.4961 & -1.473696 \\
\hline 4 & $\mathrm{C}$ & 4.984913 & -2.912579 & -1.473282 \\
\hline 4 & $\mathrm{H}$ & 4.299201 & -4.674797 & -0.448370 \\
\hline 4 & $\mathrm{C}$ & 5.6 & -3.647745 & -2.474084 \\
\hline 4 & $\mathrm{C}$ & 6.309328 & -3.006554 & -3.514089 \\
\hline 45 & $\mathrm{C}$ & 6.317096 & -1.622587 & -3.566502 \\
\hline 46 & $\mathrm{H}$ & 6.819991 & -1.107247 & -4.380631 \\
\hline 47 & $\mathrm{C}$ & 5.672973 & -0.885760 & -2.568432 \\
\hline 48 & $\mathrm{H}$ & 5.695399 & 0.198536 & -2.666319 \\
\hline 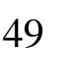 & $\mathrm{H}$ & 6.808269 & -3.586876 & -4.285012 \\
\hline
\end{tabular}




$\begin{array}{lllll}50 & \mathrm{H} & 5.633518 & -4.735832 & -2.457085 \\ 51 & \mathrm{C} & 2.462864 & 3.808408 & -0.686721 \\ 52 & \mathrm{C} & 0.192292 & 5.427274 & -1.078931 \\ 53 & \mathrm{C} & 2.267741 & 4.496149 & -1.895764 \\ 54 & \mathrm{C} & 1.504259 & 3.964903 & 0.326685 \\ 55 & \mathrm{C} & 0.372538 & 4.759033 & 0.129361 \\ 56 & \mathrm{C} & 1.141996 & 5.301216 & -2.090224 \\ 57 & \mathrm{H} & 2.988903 & 4.391708 & -2.703551 \\ 58 & \mathrm{H} & 1.624319 & 3.452219 & 1.279315 \\ 59 & \mathrm{H} & -0.371820 & 4.841755 & 0.918157 \\ 60 & \mathrm{H} & 1.001673 & 5.819184 & -3.034989 \\ 61 & \mathrm{H} & -0.692642 & 6.038967 & -1.231701 \\ 62 & \mathrm{C} & 2.757054 & -3.630792 & 1.482903 \\ 63 & \mathrm{C} & 1.239225 & -5.070561 & 3.378605 \\ 64 & \mathrm{C} & 1.878223 & -4.654968 & 1.085895 \\ 65 & \mathrm{C} & 2.858856 & -3.354550 & 2.856871 \\ 66 & \mathrm{C} & 2.103031 & -4.061919 & 3.795586 \\ 67 & \mathrm{C} & 1.129445 & -5.371349 & 2.024132 \\ 68 & \mathrm{H} & 1.764966 & -4.898763 & 0.031210 \\ 69 & \mathrm{H} & 3.536404 & -2.578644 & 3.209570 \\ 70 & \mathrm{H} & 2.194679 & -3.824237 & 4.852239 \\ 71 & \mathrm{H} & 0.460705 & -6.162581 & 1.696043 \\ 72 & \mathrm{H} & 0.654687 & -5.622361 & 4.109816 \\ 73 & \mathrm{C} & -4.608044 & -0.599708 & 0.539093 \\ 74 & \mathrm{~N} & -5.162966 & -0.142531 & -0.634260 \\ 75 & \mathrm{C} & -4.235505 & 0.644936 & -1.295115 \\ 76 & \mathrm{C} & -2.037330 & 2.120095 & -2.186888 \\ 77 & \mathrm{C} & -3.040757 & 0.714311 & -0.560879 \\ 78 & \mathrm{C} & -4.327822 & 1.321254 & -2.496849 \\ 79 & \mathrm{C} & -3.220895 & 2.058538 & -2.942552 \\ 80 & \mathrm{C} & -1.937501 & 1.438165 & -0.976049 \\ 81 & \mathrm{H} & -5.227356 & 1.287060 & -3.099780 \\ 82 & \mathrm{H} & -3.279045 & 2.597228 & -3.885825 \\ 83 & \mathrm{H} & -1.043714 & 1.504914 & -0.368927\end{array}$




\begin{tabular}{lllll}
84 & $\mathrm{H}$ & -1.195389 & 2.711772 & -2.540478 \\
85 & $\mathrm{C}$ & -6.545504 & -0.305145 & -1.022866 \\
86 & $\mathrm{H}$ & -6.977515 & 0.671626 & -1.275931 \\
87 & $\mathrm{H}$ & -7.142017 & -0.682693 & -0.181445 \\
88 & $\mathrm{C}$ & -6.710103 & -1.274247 & -2.174662 \\
89 & $\mathrm{C}$ & -7.068131 & -3.084759 & -4.291845 \\
90 & $\mathrm{C}$ & -7.433437 & -0.902780 & -3.318630 \\
91 & $\mathrm{C}$ & -6.182343 & -2.573173 & -2.102974 \\
92 & $\mathrm{C}$ & -6.357044 & -3.470473 & -3.157649 \\
93 & $\mathrm{C}$ & -7.607634 & -1.802591 & -4.372090 \\
94 & $\mathrm{H}$ & -7.866881 & 0.091476 & -3.399409 \\
95 & $\mathrm{H}$ & -5.626142 & -2.892673 & -1.222553 \\
96 & $\mathrm{H}$ & -5.937132 & -4.470703 & -3.091143 \\
97 & $\mathrm{H}$ & -8.166178 & -1.504110 & -5.255571 \\
98 & $\mathrm{H}$ & -7.203527 & -3.784787 & -5.112022 \\
99 & $\mathrm{C}$ & -1.373461 & -0.135542 & 3.779410 \\
100 & $\mathrm{H}$ & -0.948741 & -0.942938 & 3.177619 \\
101 & $\mathrm{H}$ & -2.100332 & -0.560725 & 4.476253 \\
102 & $\mathrm{H}$ & -0.576946 & 0.334877 & 4.362288 \\
103 & $\mathrm{O}$ & -5.388492 & 2.091435 & 2.791034 \\
104 & $\mathrm{C}$ & -6.213082 & 3.100195 & 2.200936 \\
105 & $\mathrm{H}$ & -6.387711 & 2.867468 & 1.144200 \\
106 & $\mathrm{H}$ & -5.724841 & 4.077097 & 2.289937 \\
107 & $\mathrm{C}$ & -7.536482 & 3.121191 & 2.940773 \\
108 & $\mathrm{H}$ & -8.028303 & 2.144785 & 2.878060 \\
$\mathrm{H}$ & -8.204278 & 3.880468 & 2.524348 \\
$\mathrm{H}$ & -7.380633 & 3.331673 & 4.004014 \\
\hline 10 & -5.267656 & -1.086902 & 1.451423
\end{tabular}

